\title{
Exploring the genetic basis of human population differences in DNA methylation and their causal impact on immune gene regulation
}

\author{
Lucas T. Husquin 1,2,3, Maxime Rotival ${ }^{1,2,3}$, Maud Fagny4, Hélène Quach ${ }^{1,2,3}$, Nora Zidane ${ }^{1,2,3}$, Lisa M. McEwen, \\ Julia L. Maclsaac ${ }^{5}$, Michael S. Kobor ${ }^{5}$, Hugues Aschard ${ }^{3}$, Etienne Patin ${ }^{1,2,3}$ and Lluis Quintana-Murci ${ }^{1,2,3^{*}}$ (D)
}

\begin{abstract}
Background: DNA methylation is influenced by both environmental and genetic factors and is increasingly thought to affect variation in complex traits and diseases. Yet, the extent of ancestry-related differences in DNA methylation, their genetic determinants, and their respective causal impact on immune gene regulation remain elusive.

Results: We report extensive population differences in DNA methylation between 156 individuals of African and European descent, detected in primary monocytes that are used as a model of a major innate immunity cell type. Most of these differences ( 70\%) are driven by DNA sequence variants nearby $\mathrm{CpG}$ sites, which account for $\sim 60 \%$ of the variance in DNA methylation. We also identify several master regulators of DNA methylation variation in trans, including a regulatory hub nearby the transcription factor-encoding CTCF gene, which contributes markedly to ancestry-related differences in DNA methylation. Furthermore, we establish that variation in DNA methylation is associated with varying gene expression levels following mostly, but not exclusively, a canonical model of negative associations, particularly in enhancer regions. Specifically, we find that DNA methylation highly correlates with transcriptional activity of 811 and 230 genes, at the basal state and upon immune stimulation, respectively. Finally, using a Bayesian approach, we estimate causal mediation effects of DNA methylation on gene expression in 20\% of the studied cases, indicating that DNA methylation can play an active role in immune gene regulation.
\end{abstract}

Conclusion: Using a system-level approach, our study reveals substantial ancestry-related differences in DNA methylation and provides evidence for their causal impact on immune gene regulation.

Keywords: Epigenetics, DNA methylation, Ancestry, Gene expression, Mediation, Immunity

\section{Background}

Individuals and populations display variable susceptibility to infectious diseases, chronic inflammatory disorders, and autoimmunity $[1,2]$. Over the last decade, it has become clear that such disparities partly result from differences in the host genetic make-up, with an increasing number of genes being associated with varying abilities to fight infections at the individual and population

\footnotetext{
* Correspondence: quintana@pasteur.fr

${ }^{1}$ Unit of Human Evolutionary Genetics, Institut Pasteur, 75015 Paris, France ${ }^{2}$ Centre National de la Recherche Scientifique (CNRS) UMR2000, 75015 Paris, France

Full list of author information is available at the end of the article
}

level $[3,4]$. Furthermore, population genetic studies have revealed that pathogen-driven selection has substantially impacted human genetic diversity $[5,6]$. Because the mortality, and thus the selective pressure, imposed by pathogens have been paramount [7], human populations had to adapt to the different pathogenic environments they encountered around the globe, and genes involved in host defense are among the functions most strongly selected for by natural selection [5, 8-11]. While substantial evidence supports this hypothesis at the genetic level, we still know little about the degree of naturally

(c) The Author(s). 2018 Open Access This article is distributed under the terms of the Creative Commons Attribution 4.0 International License (http://creativecommons.org/licenses/by/4.0/), which permits unrestricted use, distribution, and reproduction in any medium, provided you give appropriate credit to the original author(s) and the source, provide a link to the Creative Commons license, and indicate if changes were made. The Creative Commons Public Domain Dedication waiver (http://creativecommons.org/publicdomain/zero/1.0/) applies to the data made available in this article, unless otherwise stated. 
occurring epigenetic variation at the population level and how this may impact immune phenotypes.

As the immune system is the primary interface with the human pathogenic environment, the study of DNA methylation $[12,13]$ offers a unique opportunity to explore the interplay between the genome and environmental cues. DNA methylation can be affected by a range of external factors, such as nutrition, toxic pollutants, social environment, and infectious agents [14-19]. Furthermore, numerous studies have mapped DNA sequence variants associated with DNA methylation variation [20-28], i.e., methylation quantitative trait loci (meQTLs), and 20\% of the inter-individual variation in DNA methylation has been attributed to genetics $[29,30]$. DNA methylation variation has also been associated with complex traits, including aging [31], body mass index [32], various cancers $[33,34]$, obesity [35], and autoimmune and inflammatory disorders [36, 37]. Yet, most studies of human epigenome variation, both in health and disease conditions, have focused on populations of homogeneous genetic ancestry, primarily of European descent.

A few studies, however, have reported that population differences in ancestry, habitat, or lifestyle affect DNA methylation, providing an initial assessment of the contribution of genetic factors and gene-environment $(\mathrm{G} \times \mathrm{E})$ interactions to population-level epigenetic variation [38-44]. Yet, these studies investigated DNA methylation variation from virus-transformed lymphoblastoid cell lines or whole blood, so the differences observed could reflect, at least partially, epigenetic changes induced by cell immortalization or heterogeneity in blood cell composition that was not fully accounted for [45-47]. Thus, the extent of DNA methylation variation related to ancestry, and its genetic determinants, in a cellular setting relevant to immunity are far from clear.

A growing body of research has reported ancestry-related variation in terms of immune gene expression levels. Two recent studies found marked differences between individuals of African and European ancestry in their transcriptional responses to infectious challenges $[48,49]$ and showed that regulatory variants (i.e., expression quantitative trait loci, eQTLs) explain a substantial proportion of these population differences. Still, a large fraction of the variance in gene expression, both across individuals and populations, cannot be attributed to genetic factors and remains unexplained [48-55]. In this context, DNA methylation represents an additional, possible layer for variation in gene regulation [56]. The observed correlations between DNA methylation and gene expression levels can be positive and negative; in the canonical model, high levels of methylation at promoter regions are often associated with low gene expression, but elevated gene body methylation is also associated with active expression [28, 47, 57-60]. There is also increasing evidence that DNA methylation can play both passive and active roles in the regulatory interactions influencing gene expression, but the causality relationships between DNA methylation, gene expression, and genetic factors are not fully understood [19, 23, 56]. Furthermore, genetic variants associated with complex traits or diseases by genome-wide association studies (GWAS) often overlap both eQTLs and meQTLs, suggesting that disease risk can be mediated, directly or indirectly, by variation in DNA methylation [61-67].

Here, we aimed to broaden our understanding of the mechanistic links between ancestry-related differences in DNA methylation, genetic factors, and immune gene regulation. To do so, we build upon the EvoImmunoPop collection of primary monocytes originating from healthy individuals of African and European ancestry [48]. We profiled the DNA methylome of 156 donors, including 78 of each ancestry, using the high-resolution Infinium MethylationEPIC array, which captures methylation variation at more than 850,000 sites. This new dataset was combined with both genome-wide genotyping and whole-exome sequencing data, as well as with RNA-sequencing profiles from resting and stimulated monocytes with various immune stimuli, obtained from the same individuals. Such a system-level approach, integrating epigenetic, genetic, and transcriptional data, allowed us to assess the extent to which population-level variation in DNA methylation and its genetic determinants impact transcriptional activity related to immune responses.

\section{Results}

\section{Population differences in DNA methylation profiles of primary monocytes}

To assess population differences in DNA methylation of a purified innate immune cell type, we characterized DNA methylation variation at $>850,000 \mathrm{CpG}$ sites across the genome, in monocytes originating from 156 male healthy volunteers: 78 of African descent (AFB, median age $=30.9$ years) and 78 of European descent $(\mathrm{EUB}$, median age $=$ 25.9 years), all living in Belgium. Note that AFB individuals moved to Belgium between the ages of 6-45 years old (median age $=29$ years). After normalization and filtering (see "Materials and methods"), we retained a final dataset of 552,141 methylation sites in the 156 individuals (Additional file 1: Figure S1). Principal component analysis (PCA) of DNA methylation clearly separated AFB and EUB along the first two PCs, which explained together $11.6 \%$ of the total variance (Fig. 1a). At a false discovery rate $(\mathrm{FDR})=1 \%$, we identified 77,857 sites $(14.1 \%$ of the total number) that presented a significant difference between AFB and EUB in their mean level of DNA methylation, after adjusting for age and surrogate variables. When restricting our analyses to $\mathrm{CpGs}$ that presented a mean difference $>5 \%$ (measured by the $\beta$ value [68], see "Materials and methods"), we identified a total of 12,050 

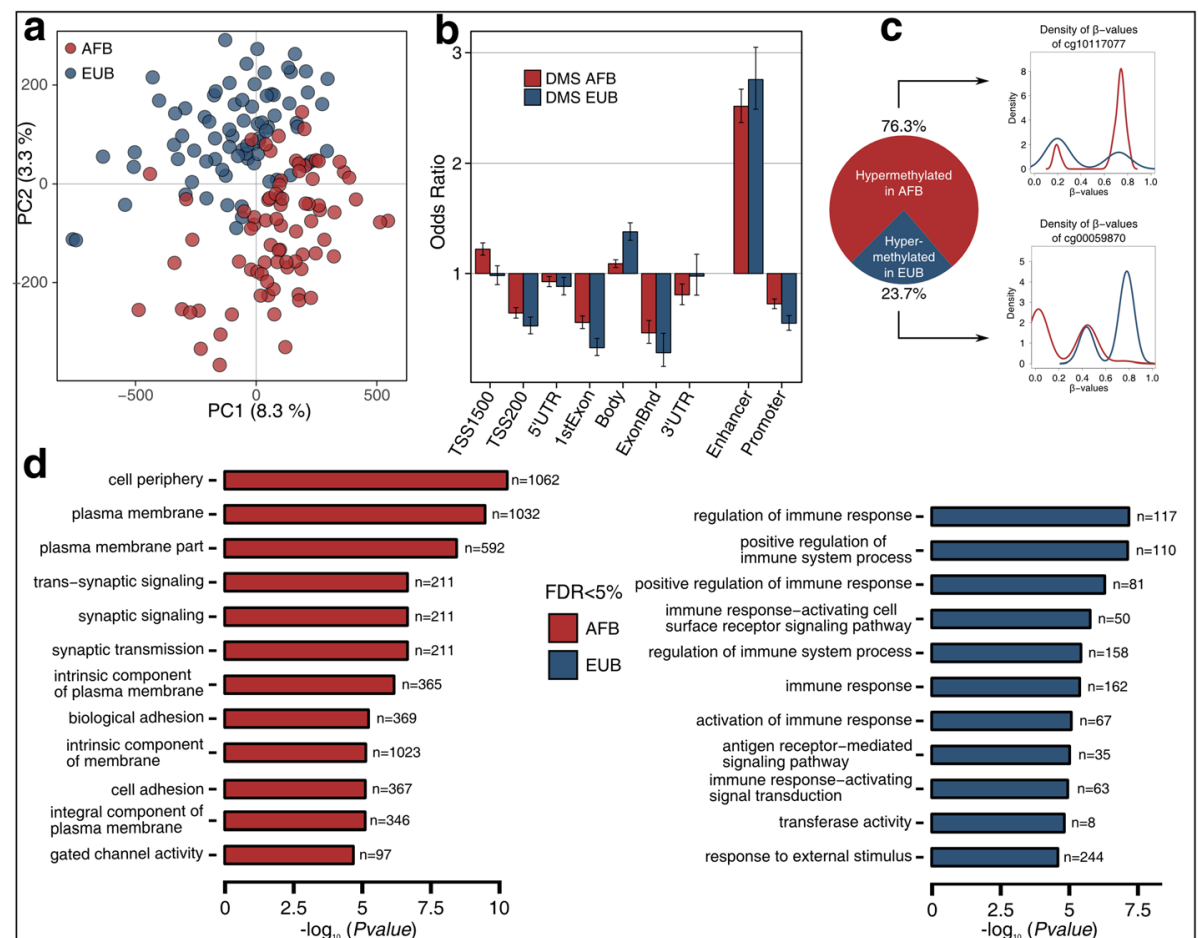

Fig. 1 Population differences in DNA methylation profiles. a Principal component analysis (PCA) of DNA methylation profiles for all 156 individuals. Red and blue circles represent African (AFB) and European (EUB) individuals, respectively. The proportions of variance explained by PC1 and PC2 are indicated. $\mathbf{b}$ Genomic location of differentially methylated sites (DMS), for CpG sites hyper-methylated in AFB (red) and in EUB (blue). Odds ratio and 95\% confidence intervals are displayed for AFB-DMS and EUB-DMS, comparing their localization in different genomic locations as provided by Illumina (TSS1500, TSS200, 5'UTR, 1stExon, Body, Exon boundaries [ExonBnd], and 3'UTR), and in enhancer and promoter regions specifically detected in monocytes by ChromHMM phase 15 (see refs. [110, 111]). Odds ratios were computed against the general distribution of the 552,141 CpGs of our dataset. c Proportion of DMS that are hyper-methylated either in AFB (red) or in EUB (blue) individuals. The density of $\beta$ values of one $\mathrm{CpG}$ site by category is given as an illustration of the population differences, with red and blue lines representing the methylation density in AFB and EUB, respectively. d Gene Ontology (GO) enrichment analyses of AFB- and EUB-DMS. For both groups, the top-GO categories reaching $5 \% \mathrm{FDR}$ are shown, together with the number of genes per category and the $\log _{10}$-transformed FDR-adjusted enrichment $P$ values

differentially methylated sites between populations (DMS) that mapped to 4818 genes. Because the age distributions of AFB and EUB individuals significantly differ (Wilcoxon $P$ value $=10^{-4}$; Additional file 1: Figure S2), and age might have a non-linear effect on DNA methylation [69], we also investigated with ANOVA the extent to which DNA methylation is non-linearly affected by age in our dataset. Our analyses showed that such effects had little to no impact on the population differences in DNA methylation detected (Additional file 2: Supplementary Note 1).

The genomic distribution of DMS, which were highly enriched in enhancer regions (odds ratio (OR) $\sim 2.6, P=1.42 \times 10^{-224}$ ), was independent of the population where hyper-methylation was observed (Fig. 1b). However, of the 12,050 DMS, $76.3 \%$ were more methylated in AFB than in EUB, with respect to the observed 54\% when considering all CpGs (Fisher's exact $P<2.2 \times 10^{-16}$ ) (Fig. 1c). The corresponding genes were enriched in Gene Ontology (GO) categories related to cellular periphery and plasma membrane
(Fig. 1d). The remaining 23.7\%, which were hyper-methylated in EUB, were enriched in sites located in genes largely associated with immune response regulation and responses to external stimulus (Fig. 1c, d; Additional file 3: Table S1). These results cannot be explained by population differences in monocyte subpopulations (i.e., CD14 high $/ \mathrm{CD} 16_{\text {neg }}$ [Classical], CD14 high $_{\text {CD16 }}$ low [Intermediate], and $\mathrm{CD} 14_{\text {low }} / \mathrm{CD} 16_{\text {high }}$ [Non-Classical]), as adding these subpopulations as covariates in the model did not alter our results (Additional file 1: Figure S3). Furthermore, we detected no $\mathrm{CpG}$ sites whose levels of methylation correlate significantly with monocyte subtypes $(\mathrm{FDR}=5 \%)$, indicating that the effects of monocyte subpopulations on DNA methylation are negligible at the epigenome-wide level. Together, these analyses reveal genes and functions that present extensive differences in DNA methylation between individuals of African and European ancestry, in the context of primary monocytes. 


\section{Genetic factors drive most ancestry-related DNA methylation variation}

We next examined the genetic determinants of the observed population differences in DNA methylation, and mapped methylation quantitative trait loci (meQTLs). We first tested for local associations between DNA methylation variation at CpGs and SNPs located within a 100-kb window (cis-meQTLs), using MatrixEQTL [70] (see "Materials and methods"). We set a 5\% FDR threshold, considering one association per CpG site and using 100 permutations $\left(P<1 \times 10^{-5}\right)$. We adjusted for age, two surrogate variables (accounting for batch effects and unknown confounders, see "Materials and methods"), and the first two PCs of the genetic data (Additional file 1: Figure S4), to account for population stratification. To detect subtle effects, we merged all individuals and included ancestry as a covariate, but simultaneously, we analyzed the two populations separately to detect putative population-specific effects. For all subsequent analyses, we present the significant results of these two approaches combined, unless otherwise indicated.

We identified 69,702 CpGs associated with at least one genetic variant in at least one population $(\sim 12.6 \%$ of all sites, referred to as meQTL-CpGs). Given that multiple linked SNPs can be associated to the same CpG, we kept the best-associated SNP for each meQTL-CpG. However, we also used a fine mapping approach [51] to detect independent SNPs associated to each CpG (see "Materials and methods"). In doing so, we detected 9826 additional meQTLs (Additional file 1: Figure S5), providing a more thorough view of the contribution of proximate genetic variants to DNA methylation variation. The median distance between a $\mathrm{CpG}$ and its associated SNP was $\sim 3.8 \mathrm{~kb}$ (Additional file 1: Figure S6), supporting the close genetic control of DNA methylation [22, 28, 41, 65]. Furthermore, we found a 2.2-fold enrichment of meQTL-CpGs in enhancers $\left(P<1 \times 10^{-326}\right)$, a trend that was even more pronounced for meQTLs associated with population differences in DNA methylation (meQTL-DMS; OR 2.8, $P=6.8 \times 10^{-317}$, Additional file 1: Figure S7).

Focusing on ancestry-related differences, we observed that $\sim 70.2 \%$ of DMS harbor a significant meQTL, with respect to the $12.6 \%$ detected genome-wide (Fisher's exact $P<2.2 \times 10^{-16}$; Fig. 2 a). These meQTLs were found to account, on average, for $\sim 58 \%$ of the observed population differences in DNA methylation (Additional file 1: Figure S8, see "Materials and methods"). Furthermore, meQTLs presented opposite effects on DNA methylation as a function of population differences in allelic frequency, i.e., a derived allele at higher frequency in Africans was generally associated with high levels of DNA methylation, while a derived allele at higher frequency in Europeans was primarily associated with low DNA methylation (Fig. 2b).
This observation provides a genetic explanation for the unbalanced patterns of hyper-methylation, observed at DMS, between Africans and Europeans (Fig. 1c).

Local meQTLs can, a priori, lead to population differences in DNA methylation following two main models: (i) the meQTL has a similar effect in both populations but present different allelic frequencies (Fig. 2c), or (ii) the meQTL is present at similar frequencies but display population-specific effects, revealing more complex interactions (Fig. 2d). We therefore investigated the population specificity of the 69,702 meQTL-CpGs detected using a model selection approach (see "Materials and methods"). We found 2868 (4.1\%) significant population-specific effects (1337 AFB-specific and 1531 EUB-specific), suggesting the occurrence of $\mathrm{G} \times \mathrm{E}$ or $\mathrm{G} \times \mathrm{G}$ effects.

\section{Ancestry-related meQTLs are enriched in associations with complex traits and diseases}

Given that a large fraction of genetic variants identified by GWAS are thought to act by affecting gene regulation [71-74], we investigated the putative functional impact of the detected meQTLs on ultimate complex phenotypes. In practice, we searched for enrichments in GWAS hits among our set of 79,528 meQTLs, correcting for linkage disequilibrium (see "Materials and methods"). Focusing on the 17 parental classes of the Experimental Factor Ontology (EFO) classification [75], we found that meQTLs were enriched in significant hits for all these functional categories (Additional file 1: Figure S9, OR $\left.\sim 2.1-5.5, P<4.1 \times 10^{-10}\right)$. Stronger enrichments were detected for meQTLs associated with population differences in DNA methylation (OR 2.79.8, $\left.P<2.9 \times 10^{-3}\right)$, in particular for phenotypes related to hematological measurements, neurological disorders, immune system disorders, inflammatory measurements, and digestive system disorders (Fig. 2e).

Because DNA methylation and meQTLs have been shown to be largely cell or tissue dependent [23, 76-81], we next searched for the specific traits that account for the signals detected at the parental category "immune system disorder", given our focus on primary monocytes. We found that meQTLs overlapped variants associated with diseases such as osteoarthritis, psoriasis, systemic lupus erythematosus, inflammatory skin disease, or type 1 diabetes (Additional file 1: Figure S10). For example, the meQTL SNP rs629953 presents markedly different frequencies between AFB and EUB (DAF AFB 7.5\% versus DAF EUB 62\%), leading to variable population-level DNA methylation at TNFAIP3 (cg06987098), and has been associated with psoriasis susceptibility [82, 83]. Together, our analyses support that complex traits and variable DNA methylation are pleiotropically associated with genetic variation $[39,60,63,64]$, but extend these 


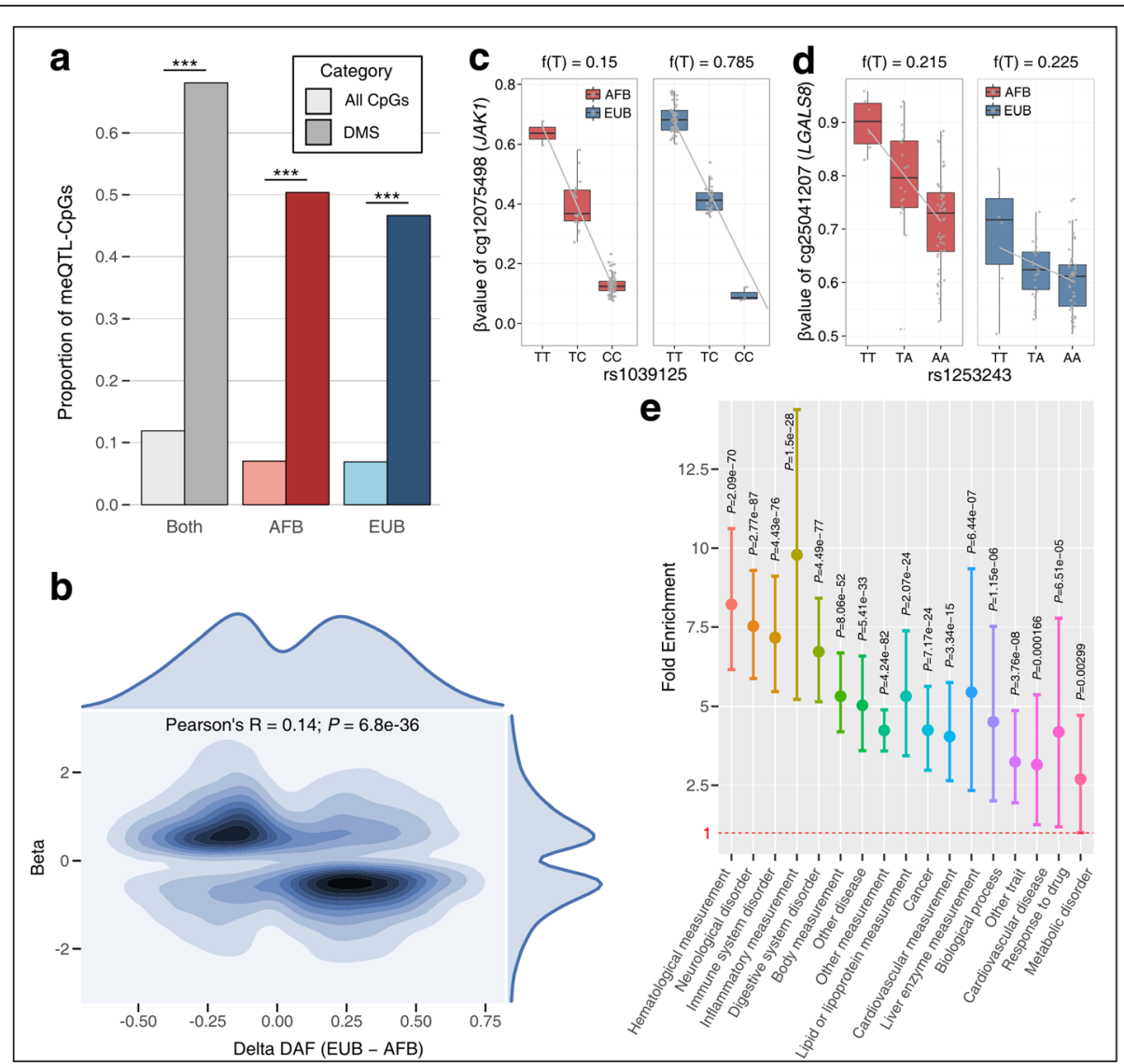

Fig. 2 Genetic control of population differences in DNA methylation levels. a Proportions of CpGs and DMS associated to genetic variants identified in the three meQTL studies: merging the two populations (gray shades), mapping in AFB only (red shades) and in EUB only (blue shades). For each mapping, proportions among all 552,141 tested CPG sites and among DMS are indicated in light and dark colors, respectively. ***Fisher's exact $P<2.2 \times 10^{-16}$. b Contour plot of meQTL effects on DMS as a function of their difference in derived allelic frequencies (DAF) between populations. For each of the 8459 DMS for which we detected at least one meQTL, we used a kernel density estimation to draw the contour plot of the effect of the derived allele of the meQTL onto methylation (beta, $Y$ axis) according to the $\triangle \mathrm{DAF}\left(\mathrm{DAF}_{\mathrm{EUB}}-\mathrm{DAF}_{\mathrm{AFB}}, X\right.$ axis). The coefficient and $P$ value of Pearson's correlation test are displayed. The marginal distribution of the two variables is displayed: top for $\triangle D A F$, and right for beta. $\mathbf{c}$, $\mathbf{d}$ Examples of meQTLs detected in this study. Boxplots represent the distribution of $\beta$ values as a function of genotype, for AFB (red) and EUB (blue) individuals. The minor allele frequency of each meQTL is presented for each population on the top. Gray lines indicate the fitted linear regression model for $\beta$ value genotype for each population. e Fold enrichment of meQTLs associated with DMS in GWAS hits. For each of the 17 parental EFO categories, the fold enrichment, the $95 \%$ confidence intervals obtained by bootstrap, and the associated $P$ values are shown

associations to variants affecting ancestry-related epigenetic variation in the context of an innate immunity cell type.

\section{Exploring the distant genetic control of DNA methylation variation}

We subsequently searched for the effects of distant genetic variants on DNA methylation variation (trans-meQTLs). To limit the burden of multiple testing, and because trans-meQTLs are enriched in cis-eQTLs for genes encoding transcription factors (TF) [65], we focused on two non-independent subsets of genetic variants: (i) the 4037 SNPs detected as cis-eQTLs for one of 600 TF-coding genes and, more generally, (ii) the 73,561 SNPs located in the vicinity $( \pm 10 \mathrm{~kb})$ of the TSS of these genes. Only associations for which the SNP-CpG distance was higher than $1 \mathrm{Mb}$ were considered, at an FDR of $5 \%\left(P<1 \times 10^{-9}\right)$. Given the generally low power to map trans-associations, we performed this analysis by considering all individuals together and including ancestry as a covariate.

We identified $133 \mathrm{CpG}$ sites associated with at least one distant SNP, for a total of 672 trans-meQTLs that involved 91 independent loci (Additional file 4: Table S2). Among these, we detected a number of hubs of distant genetic control of DNA methylation variation, including six TFs (ZNF429, CTCF, FOXI1, ZBTB25, $M K L 2$, and NFATC1) where local genetic variation was associated with at least 10 different CpGs in trans. Highlighting one pertinent example, a single genetic 
variant (rs7203742) nearby CTCF-encoding a transcriptional regulator with 11 highly conserved zinc-finger domains-controls the degree of DNA methylation at 30 CpG sites, $\sim 29.4 \%$ of all CpGs regulated in trans. Furthermore, of the 21 trans-regulated CpGs that were detected as DMS, 12 were controlled by the same CTCF variant. That this variant $(\mathrm{T} \rightarrow \mathrm{C})$ presents high levels of population differentiation (DAF AFB $24 \%$ vs. EUB $88 \%$, $F_{\mathrm{ST}}=0.59$ in the $1 \%$ of the genome-wide distribution) suggests the action of positive selection targeting the derived allele in Europeans. This observation makes of $C T C F$ not only a master regulator of DNA methylation, as previously observed [65], but also an important contributor to differences in DNA methylation between human populations.

\section{Dissecting the mechanistic relationships between DNA methylation and gene expression}

We leveraged the availability of RNA-sequencing data from the same individuals [48] to obtain new insights into the mechanistic relationships between DNA methylation and gene expression variation, in African and European individuals. We associated the levels of expression of 12,578 genes in primary monocytes with those of DNA methylation at CpGs located within 100 $\mathrm{kb}$ of their TSS, for a total of 513,536 CpG sites. Associations were considered significant if they passed a $P$ value threshold determined using 100 permutations $\left(\mathrm{FDR}=5 \%, P<5 \times 10^{-5}\right)$ (see "Materials and methods").

We identified 1666 CpGs whose levels of DNA methylation were associated with gene expression (eQTMs), for a total of 811 genes (eQTM-genes) associated with at least one $\mathrm{CpG}$ in one population group (Additional file 5: Table S3). The KEGG pathways associated with eQTM-genes contained a large number of immune-related pathways, providing a link between DNA methylation and gene expression in the context of immunity (Fig. 3a). When investigating the population specificity of the 811 eQTMs (see "Materials and methods"), we detected 93 significant population-specific effects (43 AFB-specific and 50 EUB-specific). The majority of these cases (80 out of 93) corresponded to genes whose eQTMs were also under genetic control, suggesting, again, the occurrence of $\mathrm{G} \times \mathrm{G}$ or $\mathrm{G} \times \mathrm{E}$ interactions.

Based on current genomic annotations, eQTMs were mostly negatively correlated to gene expression $(69.5 \%$ vs. $30.5 \%$, see also refs. [23, $28,65,84,85])$. Negatively correlated sites were strongly enriched in enhancers (OR 2.6, $P=6.6 \times 10^{-59}$ ) (Fig. 3b), highlighting their major role in transcriptional regulation [86-88]. In addition, we found a slight excess of negative associations in promoters (OR $\left.\sim 1.2, P=1.8 \times 10^{-2}\right)$ and nearby TSS (TSS1500) (OR 1.4, $\left.P=7.2 \times 10^{-13}\right)$, as expected following the canonical model. Conversely, positive associations were enriched in sites located nearby UTRs, particularly 3 '-UTR (OR $\sim 1.8, P=8.4 \times 10^{-5}$ ) [89], but depleted in sites located in promoters (OR $\sim 0.6, P=1.1 \times 10^{-4}$ ) (Fig. 3b). Furthermore, we found that eQTMs were strongly enriched in DMS (OR 11.8, $\left.P<1.93 \times 10^{-216}\right)$ and, importantly, in meQTLCpGs (OR $\sim 33.2, P<1 \times 10^{-326}$ ) (Fig. 3c). Together, these observations indicate that DNA methylation variation, in particular at sites that are differentially methylated across populations (DMS), is much more likely to be under genetic control when associated with gene expression differences (eQTMs), than random CpG sites.

\section{Exploring the underlying causality between regulatory loci and gene expression}

Because the respective roles of genetic and epigenetic factors in transcriptional regulation are not fully understood [56], we next mapped eQTLs (FDR $=5 \%$, see "Materials and methods") to identify the cases where DNA methylation, gene expression, and genetic variants show significant associations between all pairs (Additional file 1: Figure S11). We thus obtained 552 trios, each of them consisting of one gene, one to various $\mathrm{CpGs}$ and one to various SNPs (containing 68.1\% of the genes detected in the eQTM mapping). This suggested potential, causal relationships between these variables-a latent, though challenging, question in epigenetics. To infer causality between regulatory loci (i.e., eQTMs and eQTLs) and gene expression variation for these specific trios, we first used an elastic net model to build two intermediate variables measuring (i) DNA methylation variability attributable to genetics for the trios presenting more than one SNP and (ii) gene expression variability attributable to DNA methylation for the trios presenting more than one CpG (see "Materials and methods").

We used a Bayesian approach [90] to assess potential causal effects of a mediating variable $M$ (DNA methylation) on the relationship between an independent variable $X$ (genetics) and a dependent variable $Y$ (gene expression) [91]. When comparing the performance of this method with that of an approach based on partial correlations, using simulated data and various genomic scenarios, we found similar results between the two approaches in terms of sensitivity and specificity (Fig. 4a, b; Additional file 1: Figure S12; see "Materials and methods"). We then ran the mediation analysis on each trio, adjusting for regular covariates (age and surrogate variables), but also for the fourth and second PCs of gene expression and DNA methylation, respectively. The latter covariates were added because they likely capture potential confounding factors inducing correlation between DNA methylation and expression, which would violate the assumption of the causal inference model (Additional file 1: Figure S13). Note that reverse 


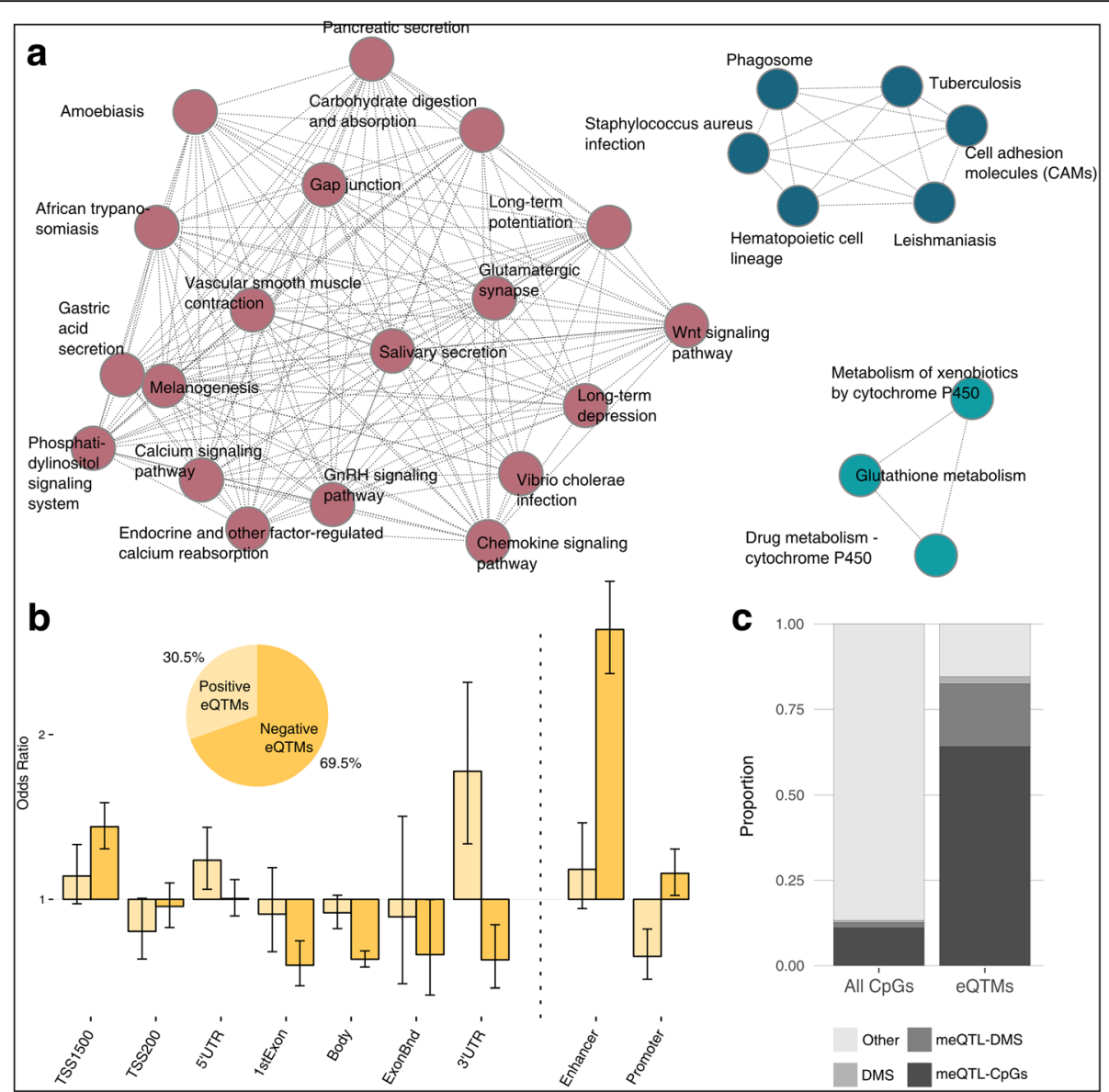

Fig. 3 Correlations of DNA methylation with gene expression. a Networks of KEGG pathways of genes detected in the eQTM mapping. b Genomic location of eQTMs, for positively and negatively associated CpG sites (light and dark yellow, respectively). Odds ratio were computed against the general distribution of the 552,141 CpGs from our dataset. The distribution of eQTMs according to the direction of their effect on gene expression is shown. c Proportions of different groups of CpG sites in all tested sites (left panel) and among the detected eQTMs (right panel)

causation was found to be unlikely in our experimental setting and was thus not considered in our analyses (Additional file 2: Supplementary Note 2).

At $\mathrm{FDR}=5 \%$, we identified 165 genes where the genetic control of expression levels was mediated by DNA methylation (i.e., $\alpha \times \beta$ was significantly different from zero, Fig. 4a), in at least one population. Remarkably, in 66 of these cases, mediation occurred through $\mathrm{CpG}$ sites that are differentially methylated across populations (DMS) (Additional file 6: Table S4). The proportion of mediated genes whose expression was positively and negatively correlated to DNA methylation was similar, ranging from 26 to 31\% (Fig. 4c). Expectedly, we found that, among mediated genes, DNA methylation explained a significantly higher proportion of the variance of gene expression than genetics (mean $R^{2}=23.4 \%$ versus $15.4 \%$, respectively; Wilcoxon $P=3.3 \times 10^{-11}$ ), in contrast with the 387 non-mediated cases where we observed the opposite trend (Wilcoxon $P=7.8 \times 10^{-37}$ ) (Fig. 4d).
We also found that $\mathrm{CpG}$ sites mediating gene expression were preferentially located in enhancers $(\mathrm{OR} \sim 2.5$, $\left.P=4.0 \times 10^{-21}\right)$, highlighting again the major role of these regions in epigenetic regulatory mechanisms [92-94]. These CpGs were depleted in promoters $(\mathrm{OR} \sim 0.7$, $\left.P=1.4 \times 10^{-2}\right)$, which were otherwise enriched in non-mediating CpGs (OR $\left.\sim 1.3, P=5.9 \times 10^{-3}\right)$. Notably, $86.6 \%$ of mediating CpGs fell directly into a TF-binding site (TFBS), with respect to the expected $76.9 \%$ at the genome-wide level (OR $\sim 1.9$, Fisher's exact $P=8.64 \times 10^{-7}$ ). This result suggests that DNA methylation might actively regulate transcriptional activity through the modulation of TF binding, a hypothesis that requires experimental validation.

Interestingly, among mediated cases, we found key genes of the immune response, such as NLRP2, RAI14, NCF4, or ICAM4, and genes with functions related to transcriptional activity, encoding zinc-finger proteins (Additional file 6: Table S4). This suggests a more extensive role of DNA methylation in regulating gene 


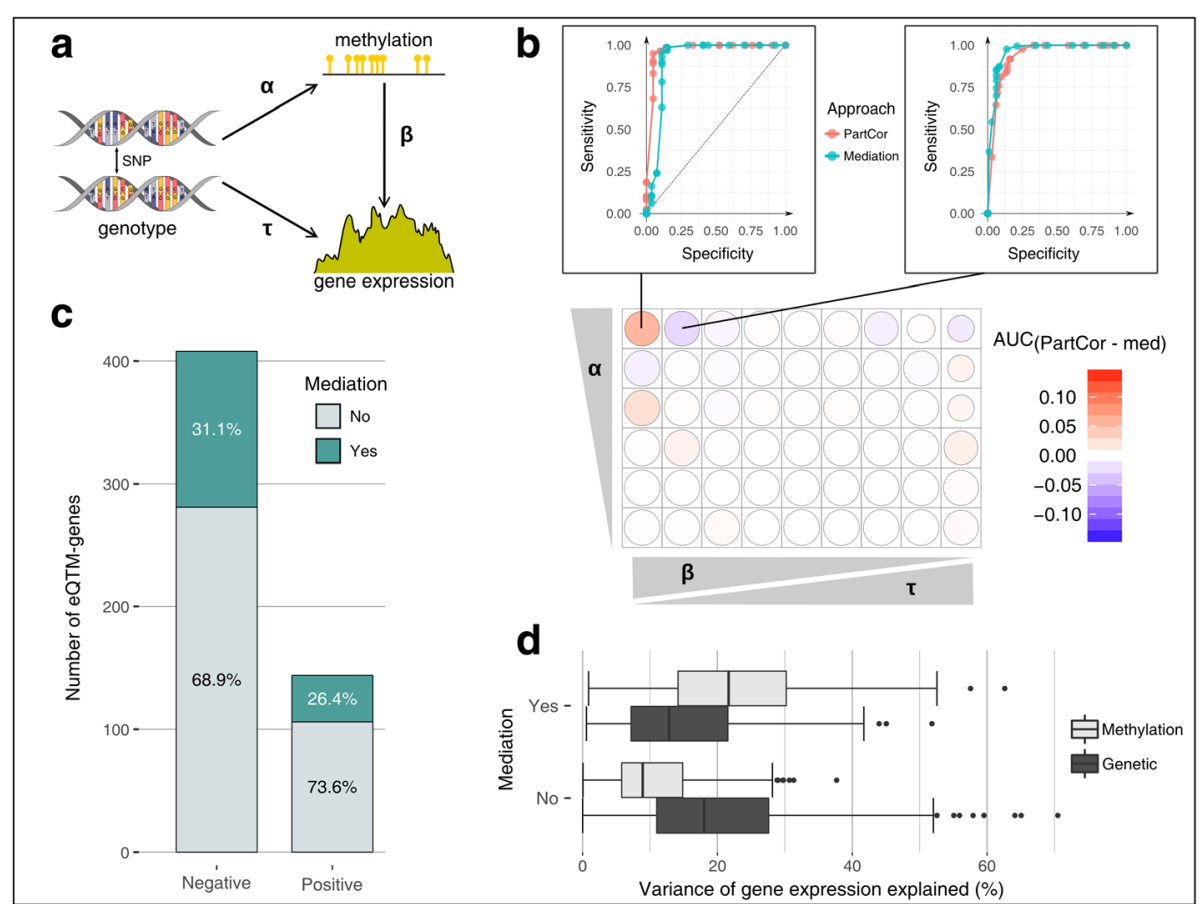

Fig. 4 Inference of the causal effects of DNA methylation on gene regulation. a Representation of a simulated scenario, with the three varying parameters $(\alpha, \beta$, and $\tau)$. $\mathbf{b}$ Comparison of the mediation analysis (med) with a partial correlation approach (PartCor) using a range of different simulated parameters for $a(0.3-0.8), \beta(0.9-0.1)$, and $\tau(0.1-0.9)$. Note that the parameter range simulated for $\beta$ and $\tau$ was adjusted so that we kept $75 \%$ of the variance unexplained (random noise parameter $\gamma=0.25$ ). The difference of the area under the curve (AUC) between the two approaches is represented with different shades of red and blue. The sizes of the circles are proportional to the mean AUC of the two approaches. Two examples of the ROC curves are shown in the upper part of the figure. c Number of mediated and non-mediated eQTM-genes for negative and positive associations between DNA methylation and gene expression. The percentages of these two categories are also indicated. $\mathbf{d}$ Proportion of variance of gene expression explained by DNA methylation (light gray) and genetics (dark gray), in mediated and non-mediated cases

expression than the local associations described here, through the regulation of DNA-binding protein activity.

\section{Impact of immune perturbation on genetic and epigenetic interactions}

Finally, we sought to understand how DNA methylation variation at the basal state affects transcriptional responses to immune activation. We used RNA-sequencing data, obtained from the same individuals, after exposure to various stimuli: LPS activating TLR4 and Pam3CSK4 activating TLR1/2, both pathways sensing bacterial components, R848 activating TLR7/8, predominantly sensing viral nucleic acids, and influenza A virus (IAV) [48]. We then mapped response-QTMs (reQTMs) using fold changes in gene expression between non-stimulated and stimulated states, for all genes expressed in either condition (see "Materials and methods").

We found 230 unique genes whose response to immune activation was associated with DNA methylation in at least one condition; most associations were context-specific, with only 7 genes detected in all conditions (Fig. 5a; Additional file 5: Table S3). Furthermore, a 2.5-fold increase was observed in the number of reQTM-genes detected upon activation with viral-stimuli (R848 and IAV; 197 unique genes) with respect to those detected for bacterial ligands (LPS and Pam3CSK4; 78 unique genes) (Fig. 5a). For example, we detected a reQTM upon R848 stimulation for CARD9 in EUB and CD1D upon IAV infection in AFB, both genes known to play an important role in host defense (Fig. 5b, c). Despite reQTMs and eQTMs present a similar genomic distribution (Additional file 1: Figure S14), we observed an important shift towards positive associations between DNA methylation and transcriptional responses, in particular to TLR ligands (Fig. 5d). This shift was mainly accounted for by reQTMs that present the strongest associations between DNA methylation and gene expression in the non-stimulated condition (Additional file 1: Figure S15), corresponding to 109 genes ( $47 \%$ of the total). This contrasts with the canonical model of negative associations primarily observed at reQTMs presenting the strongest associations at the stimulated state, corresponding to 131 genes (57\% of the total). Note that 10 genes were associated with reQTMs of both groups. 


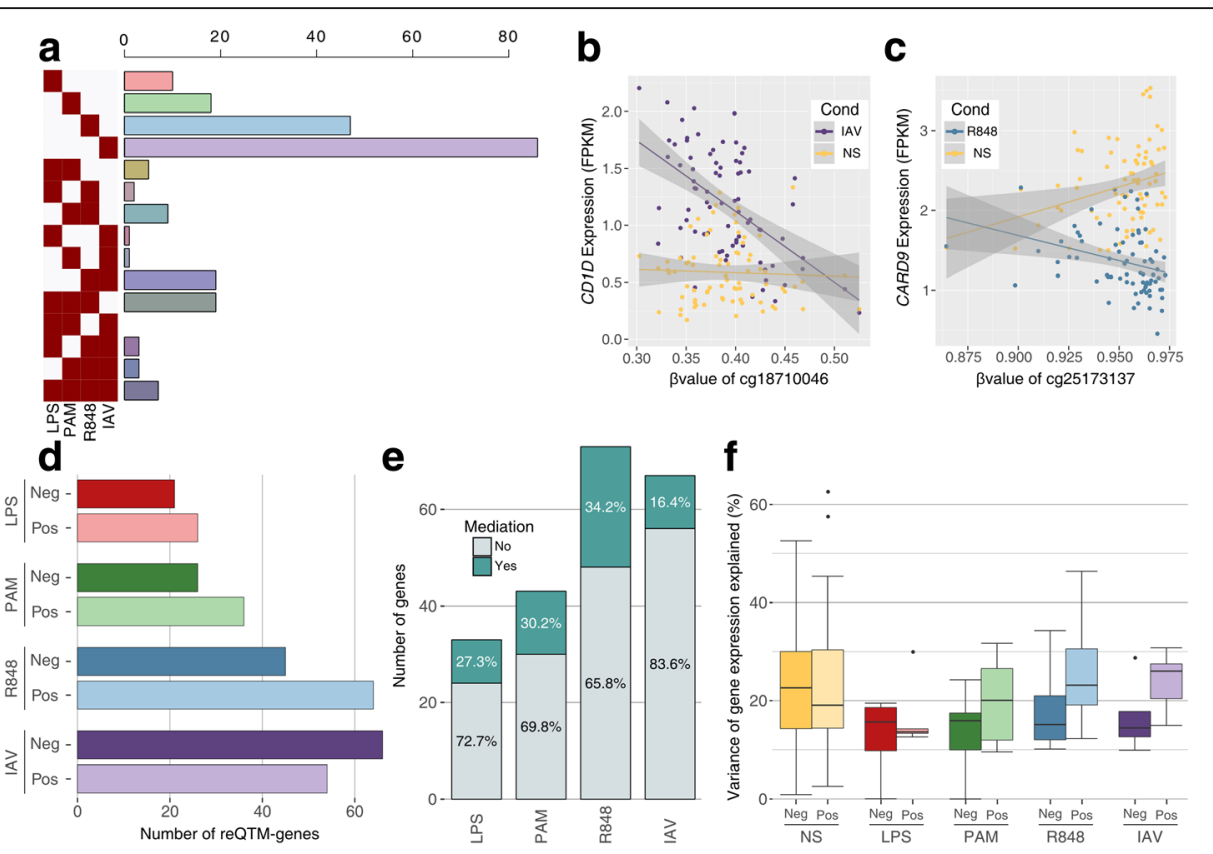

Fig. 5 Effects of DNA methylation on transcriptional responses to immune stimulation. a Number of genes harboring reQTMs in single conditions or combinations of stimulations. b, c Examples of reQTMs detected in this study. Lines indicate the fitted linear regression model, and gray shades the $95 \%$ confidence intervals of these models. b The distribution of the expression values of CD1D at the non-stimulated (yellow) and after IAV infection (purple) is plotted as a function of $\beta$ values, for AFB individuals only. $\mathbf{c}$ The distribution of the expression values of CARD9 at the non-stimulated (yellow) and upon R848 stimulation (blue) is plotted as a function of $\beta$ values, for EUB individuals only. $\mathbf{d}$ Number of reQTMgenes by condition and according to the direction of their association with DNA methylation. e Number of mediated and non-mediated reQTMgenes per stimulation condition. The percentages of these two categories for each condition are also indicated. $\mathbf{f}$ Proportion of variance of gene expression explained by DNA methylation, among negative (dark colors) and positive (light colors) associations, in mediated cases

To explore causal mediation effects of DNA methylation in the context of immune activation, we mapped response-QTLs (see "Materials and methods"). Following our previous rationale (Additional file 1: Figure S11), we identified 141 trios (61.3\% of the 230 reQTM-genes, Additional file 6: Table S4). At FDR $=5 \%$, we detected 40 genes (28.4\%) where the genetic control of their transcriptional response was mediated by DNA methylation (Fig. 5e). Although non-significant, we found a higher proportion of mediation for genes whose response was positively associated with DNA methylation, as compared to negative associations, in particular for viral challenges $(\mathrm{OR} \sim 2.0$; Fisher's exact $P=0.33$ ) (Additional file 1: Figure S16). Among mediated genes in the viral conditions, the proportion of gene expression variance explained by DNA methylation was higher for positive than for negative associations, again at odds with the non-stimulated condition (Fig. 5f). More generally, our analyses illustrate the value of mapping reQTMs and studying the underlying patterns of causality, to uncover mechanisms that might explain disparities in the way individuals and populations respond to immune activation.

\section{Discussion}

Our population epigenetic results, obtained in the setting of an innate immunity cell population, demonstrate extensive differences in DNA methylation profiles between two populations that differ in their genetic ancestry but share the same present-day environment. Such population differences were observed at the epigenome-wide level (explaining $\sim 12 \%$ of the total variance in DNA methylation) and involved 12,050 sites that were mostly located in genes with functions related to cell periphery or immune response regulation. Previous studies have searched for ancestry-related differences in DNA methylation in various human populations and cell types [16, 38-41, 43, 95]. Although comparisons across studies are complicated by differences in experimental settings and statistical thresholds used to detect ancestry-associated CpG sites, these range from 299 between Caucasian- and Asian/mixed-descent individuals living in Canada [16] to 36,897 between European CEU and African YRI [39]. An interesting insight that can be drawn from our analyses is that genes involved in the activation and regulation of immune responses tend to present higher levels of DNA methylation in individuals of European ancestry, with respect to those of African 
ancestry, mostly owing to genetic control. That up to $16 \%$ of immune-related genes that are hyper-methylated in Europeans are also differentially expressed between populations [48] could provide a mechanistic explanation for the ancestry-related differences in transcriptional responses to bacteria reported in macrophages, where European ancestry is associated with lower inflammatory responses [49].

Although variation in past environmental exposures and socioeconomic factors may contribute to population differences in DNA methylation, we found that $70 \%$ of differentially methylated sites between African and European ancestry groups were associated with at least one meQTL. This indicates that population differences in DNA methylation are mostly driven by DNA sequence variants $[38,40-42]$. In some cases, a single genetic variant can account for important population differences at multiple CpG sites, as attested by the trans-meQTL we detected at CTCF, whose local genetic variation has been shown to alter distant DNA methylation patterns in whole blood [65]. We show that a CTCF variant (rs7203742) regulates DNA methylation of 30 distant CpGs, $40 \%$ of which are differentially methylated between populations. We also found that all CTCF trans-regulated CpGs fall within a TFBS, confirming our initial hypothesis about the mechanism by which a genetic variant might alter DNA methylation at a distant CpG site. Interestingly, 9 out of the 30 CTCF trans-regulated CpGs fall within a TFBS of $C T C F$, while the remaining 21 fall within a TFBS specific to other TFs such as YY1, ESR1, or ZNF143. This observation is consistent with a model of pioneer transcription factor activity [96] and suggests that CTCF acts as a pioneer factor that will generate changes in chromatin state that, in turn, will become accessible for binding of secondary factors.

At the genome-wide level, we find that the quantitative impact of DNA methylation on gene expression variation is lower than that reported by some previous studies, possibly reflecting differences in experimental settings and statistical power (e.g., cell types and sample sizes) [23, 65, 84, 89]. For example, a study of 204 healthy newborns detected substantial variation across tissues in the number of genes whose expression levels were associated with DNA methylation, ranging from 596 in fibroblasts to 3838 in T cells [23]. We detected, at the non-stimulated state, 811 eQTM-genes $(6 \%$ of the total number of expressed genes), a figure that drops to 230 for reQTM-genes across stimulation conditions. However, a limitation of our study is that we measured DNA methylation at the basal state, while gene expression was obtained after $6 \mathrm{~h}$. Studies including a more comprehensive range of epigenetic marks obtained at different time points-in different cell types and tissues originating from individuals of various ancestries-are needed to more precisely understand the interplay between these regulatory elements and quantify their respective roles in the regulation of transcriptional activity.

The detected eQTMs were found to be drastically enriched in genetic control (OR $\sim 33.2, P<1 \times 10^{-326}$, Fig. 3c), which highlights the coordinated action of genetic and epigenetic factors in driving gene expression variation but raises questions about the causal role of DNA methylation [56]. Despite cautious interpretation of causality in mediation analyses is required [97], our analysis provides a first estimate of the potential direct role of DNA methylation in regulating transcriptional activity, in both resting and stimulated monocytes. At the non-stimulated state, we find that $\sim 20 \%$ of eQTM-genes show evidence of a causal mediation effect of DNA methylation. Although a similar extent of mediation was found upon immune stimulation $(\sim 17 \%)$, we detected specific patterns upon treatment with viral challenges, where a higher occurrence of positive associations was observed among mediated cases. These findings mostly reflected cases where high levels of DNA methylation were associated with low gene expression in the non-stimulated condition, thus requiring stronger responses to reach high levels of gene expression upon cell perturbation. These trends suggest a major, direct, and context-specific role of DNA methylation in the regulation of immune responses, whose complexity requires further investigation.

Finally, we found that meQTLs, in particular those associated with ancestry-related differences, are enriched in GWAS hits related to immune disorders. This suggests that DNA methylation has an important impact on the cellular activity of monocytes and ultimately affect phenotypic outcomes. Nonetheless, a large fraction of the variance of DNA methylation and gene expression remains unexplained. Additional work is needed to quantify the relative impact of genetic, epigenetic, environmental, and lifestyle factors in driving variation of DNA methylation and gene expression, both in resting and stimulated cells. Furthermore, although the causal mediation analyses presented in this study reinforce the notion that DNA methylation can play a direct role in regulating gene expression in humans $[23,98]$, monitoring the kinetics of variation in DNA methylation and gene expression after exposure to different infectious agents will broaden our understanding of the interplay between these molecular phenotypes and their impact on endpoint phenotypes.

\section{Conclusion}

Our study reveals extensive variation in DNA methylation profiles between individuals and populations, with ancestry-related differences being mostly explained by genetic variation. It also suggests that DNA methylation can have a direct, causal impact on the transcriptional 
activity of primary monocytes, providing new insight into the nature of the host factors that drive immune response variation in humans.

\section{Materials and methods}

\section{Sample collection and monocyte purification}

The EvoImmunoPop collection consists of 156 individuals (males between 20 and 50 years old, mean 31.5 years old) from two different ancestries (78 of European and 78 of African descent), who were recruited at the Center for Vaccinology from the Ghent University Hospital (Ghent, Belgium) [48]. For each participant, $300 \mathrm{ml}$ of whole blood was collected into anticoagulant EDTA-blood collection tubes and peripheral blood mononuclear cells (PBMCs) were purified using Ficoll-paque density gradients (\#17-1440-03, GE Healthcare). Monocytes were positively selected from purified PBMCs using magnetic CD14 microbeads (\#130-050-201, MiltenyiBiotec), as per manufacturer's instructions. All samples had a monocyte purity higher than $90 \%$ with a mean value of $97 \%$.

\section{DNA methylation profiling and data normalization}

Genomic DNA was extracted from the monocyte fraction using a phenol/chloroform protocol followed by ethanol precipitation. The DNA was then bisulfite converted, and BC-DNA was then processed using the Illumina Infinium MethylationEPIC BeadChip Kit (Illumina, San Diego, CA) to obtain the methylation profile of each individual at more than $850,000 \mathrm{CpG}$ sites genome-wide.

In total, 184 samples were hybridized with the EPIC array, including 172 unique samples and 12 technical replicates. We removed any technically unreliable probes: (i) potentially cross-hybridizing probes $(83,635$ probes), (ii) those located on the $\mathrm{X}$ and $\mathrm{Y}$ chromosomes (17,229 probes), and (iii) probes overlapping SNPs that present a frequency higher than $1 \%$ in at least one of the studied populations (206,998 probes). These SNPs were chosen based on our own genotyping dataset, as well as on the 1000 Genomes project [99]. To control for the quality of the probes and samples, we filtered out individuals with $>5 \%$ of probes associated with a detection $P$ value $>10^{-3}$, and then, probes with a detection $P$ value $>10^{-3}$ in one or more individuals (6833 probes). Following this filtering process, 552,141 of the original 866,836 sites on the array were retained.

We calculated methylation levels from raw data, using the R Bioconductor lumi package [100]. Given that the $M$ value has been shown to provide better detection sensitivity than $\beta$ values at extreme levels of modification [68], we used the $M$ value to run all statistical analysis unless otherwise stated. Note that in some instances of the text and figures, $\beta$ values are reported for ease of clarity and interpretation. $M$ values were then adjusted for background noise with the normal-exponential using out-of-band probes (noob) from the $\mathrm{R}$ Bioconductor minfi package [101]. Next, normalization for color bias was performed using lumiMethyC with the "quantile" method, and for methylated/unmethylated intensity variation using the lumiMethyN with the "ssn" method [100]. Finally, we corrected for technical differences between type I and type II assay designs, by performing beta-mixture quantile normalization [102]. To correct for known batch effects and potential hidden confounders, we used the sva function from the sva Bioconductor package [103] with age as a variable of interest. Additionally, five EUB samples were removed because they presented an excess of hemimethylated sites, leaving $89 \mathrm{EUB}$ and 78 AFB samples. To obtain equal power in the two studied populations, we down-sampled the European group to 78 samples by randomly removing 11 EUB samples, for an overall final cohort of 156 individuals.

\section{Extraction of differentially methylated sites (DMS)}

To detect CpG sites presenting statistically different levels of DNA methylation between AFB and EUB, we fitted a linear regression model for each CpG site: $M$ value population + age + two surrogate variables + error, and next applied an empirical Bayes smoothing to the standard errors using the R Bioconductor limma pipeline [104]. $P$ values were adjusted using the Benjamini and Hochberg method. DMS were extracted using a threshold of adjusted $P$ value $(<0.01)$ and a difference in the mean $\beta$ value of each population $|\Delta \beta|>5 \%$.

\section{Mapping of methylation quantitative trait loci (meQTLs)}

All individuals were genotyped for a total of 4,301,332 SNPs on the Illumina HumanOmni5-Quad BeadChips and went through whole-exome sequencing with the Nextera Rapid Capture Expanded Exome kit, on the Illumina HiSeq 2000 platform, with 100-bp paired-end reads. Details of the processing of genotyping and whole-exome sequencing data, together with imputation using the 1000 Genomes Project imputation panel [99], are reported in ref. [48]. For the meQTL mapping, we filtered out SNPs with a minor allele frequency $<5 \%$ in the populations studied and kept a final dataset of 10,278,745 SNPs (i.e., corresponding to the merged genotyping and whole-exome sequencing dataset after imputation; 8,913,090 SNPs in Africans and 6,178,808 SNPs in Europeans). Age, PC1 and $\mathrm{PC} 2$ of the genotype matrix, and two surrogate variables, as identified with the sva $\mathrm{R}$ package, were used as covariates in the linear model.

We mapped meQTLs using the statistical framework implemented in the MatrixEQTL $\mathrm{R}$ package [70]. For local associations (i.e., distance SNP-CpG $\leq 100 \mathrm{~kb}$ ), we performed two independent mappings using (i) the direct linear model from the MatrixEQTL pipeline and (ii) a Kruskal-Wallis rank test. Associations were considered 
significant when passing the 5\% FDR threshold in both mappings. Two models were considered: merging all individuals and including a binary variable adjusting for ancestry or keeping the two populations separately. To detect all possible independent SNPs regulating methylation at a single CpG site in cis, we regressed out genotypes of all primary cis-meQTLs and then performed cis-meQTL mapping on the regressed methylation data to find secondary cis-meQTLs. We repeated this process in a stepwise fashion until no additional independent cis-meQTLs were detected. This allowed us to refine our local meQTL mapping by detecting all possible independent SNP-CpG associations.

For distant, trans-acting associations (i.e., distance between SNP and $\mathrm{CpG} \geq 1 \mathrm{Mb}$ or on different chromosomes), we restricted our analysis to SNPs located in the vicinity of transcription factor (TF) coding genes, to limit the burden of multiple testing. Specifically, we selected (i) all SNPs located less than $10 \mathrm{~kb}$ to the TSS of any expressed TF in our dataset and (ii) SNPs detected as cis-eQTLs for these TFs. For each SNP, we only investigated $\mathrm{CpG}$ sites that mapped at least $1 \mathrm{Mb}$ from the SNP or located on other chromosomes, using a Kruskal-Wallis rank test.

For both cis- and trans-meQTLs, FDR was computed by mapping meQTLs on 100 datasets with the $M$ values permuted within each population. We then kept, after each permutation, the most significant $P$ value per $\mathrm{CpG}$ site, across populations (probe-level FDR). Finally, we computed the FDR associated with different $P$ value thresholds for cis or trans, and subsequently selected the $P$ value threshold that provided a 5\% FDR: $P=1 \times 10^{-5}$ and $P=1 \times 10^{-9}$ for cis- and trans-meQTLs, respectively.

\section{Investigating the genetic basis of population differences in DNA methylation}

We aimed at identifying the proportion of the population differences in DNA methylation that was accounted for by genetic variability. To do so, for the 8459 DMS that were associated with at least one meQTL, we computed the following ratio:

$$
\text { ExpDiff }=\frac{\beta \times \Delta D A F}{\Delta M e t h}
$$

with $\beta$ reflecting the effect of the derived allele of the meQTL on methylation, $\triangle \mathrm{DAF}$ the difference in allelic frequencies between Europeans and Africans $\left(\mathrm{DAF}_{\mathrm{EUB}}-\right.$ $\left.\mathrm{DAF}_{\mathrm{AFB}}\right)$, and $\triangle$ Meth the observed difference in the mean levels of DNA methylation between European and African individuals $\left(\overline{\text { Meth }_{E U B}}-\overline{\text { Meth }_{A F B}}\right)$.

Note that this ratio is not bound to [0:1], as the effect of genetics onto the overall population differences in DNA methylation can be counteracted by opposite effects of independent origins (e.g., environmental factors or non-detected independent genetic effects).

\section{Detecting population-specific meQTLs}

We aimed at refining our meQTL mapping by detecting population-specific meQTL effects (i.e., SNPs present at similar frequencies in both populations but having different effect sizes on DNA methylation between populations). To do so, we used a Bayesian model selection approach to identify specific and shared effects for each of the 69,702 CpGs that we detected as being associated with at least one genetic variant. Specifically, for each CpG-SNP pair, we computed the likelihood of three models:

$$
\begin{aligned}
& \operatorname{lm}(\text { Meth } \sim S N P+\text { Pop }) \\
& \operatorname{lm}\left(\text { Meth } \sim S N P_{E U B}+\text { Pop }\right) \\
& \operatorname{lm}\left(\text { Meth } \sim S N P_{A F B}+\text { Pop }\right)
\end{aligned}
$$

with $\mathrm{SNP}_{\mathrm{EUB}}$ coded $0,1,2$ in EUB individuals and 0 in AFB individuals, and $\mathrm{SNP}_{\mathrm{AFB}}$ coded $0,1,2$ in AFB individuals and 0 in EUB individuals. We next calculated the posterior probability of each model assuming that all models are equally likely a priori. We then set a threshold of 0.9 to consider one of the models as supported by the data. Thus, a meQTL is classified as EUB-specific if the posterior probability of model (ii) is higher than 0.9 , or AFB-specific if the probability of model (iii) is higher than 0.9.

\section{GWAS enrichment analyses}

We used the NHGRI GWAS catalog [105] to first select all significant SNPs that were significantly associated with a complex trait or disease at a $P<1 \times 10^{-8}$. Using this set of GWAS hits, we next extracted all SNPs in LD with each of these hits $\left(R^{2}>0.8\right)$ and classified the resulting final set of 166,248 SNPs according to their parental Experimental Factor Ontology (EFO) term [75].

We then selected all meQTLs in our dataset that passed the $P$ value threshold corresponding to FDR 5\% in our initial mapping, and filtered out meQTLs that were in LD $\left(R^{2}>0.8\right)$ keeping one SNP per independent loci $(56,574$ independent SNPs). For the resampling set, we considered all SNPs that were initially used for the meQTL mapping and pruned them for $\mathrm{LD}\left(R^{2}>0.8\right)$, yielding a final set of 921,466 SNPs. Resampling was performed using bins of allelic frequencies at intervals of $5 \%$.

Finally, we tested for fold enrichments of meQTLs in GWAS hits, for each of the 17 parental EFO categories [75]. The fold enrichment was calculated by comparing the number of LD pruned-meQTLs that were found to correspond to GWAS hits (or were in LD with GWAS hits) with the expected number estimated through 10,000 resamples. $P$ values associated to the fold enrichment were calculated by fitting a normal distribution to the empirical 
distribution of our 10,000 resampled sets of SNPs. Confidence intervals were computed using 10,000 resamples by bootstrap. The same procedure was applied when searching for enrichments of meQTLs specifically in GWAS hits related to the 268 traits of the "Immune system disorder" EFO parental term.

\section{Expression quantitative trait methylation (eQTM) analysis}

To identify associations between DNA methylation levels and gene expression of nearby genes, we leveraged RNA-sequencing data obtained from the same individuals, both at the non-stimulated state (NS) and in response to four immune stimuli [48]. Briefly, RNA-sequencing was performed on the Illumina HiSeq2000 platform with 101-bp single-read sequencing with fragment size of around $295 \mathrm{bp}$, and outputs of around 30 million single-end reads per sample were obtained. A total of 763 RNA-sequencing samples from our filtered dataset of 156 donors were analyzed for gene expression profiling, including 156, 151, 153, 148, and 155 samples for the NS, LPS, Pam3CSK4, R848, and IAV conditions, respectively. Details of cell culture, immune stimulation conditions, and RNA-seq processing can be found in ref. [48].

Using the RNA-sequencing data from the NS condition, we mapped eQTMs (i.e., CpGs whose variation is associated with gene expression) in a window of $100 \mathrm{~kb}$ around the TSS of each gene (12,578 expressed genes in primary monocytes). The associated $P$ values and the coefficients of correlation between methylation profiles and gene expression were obtained using Spearman's rank correlation. FDR was computed by mapping eQTMs on 100 datasets with the $M$ values permuted, and kept, after each permutation, the most significant $P$ value per gene (gene-level FDR). We selected the $P$ value threshold that provided a 5\% FDR $\left(P=5 \times 10^{-5}\right)$.

We also mapped eQTMs in the context of the response to the various stimulations, namely response-QTMs (reQTMs). To do so, the same procedure explained above for the eQTM mapping was followed, using the fold change of expression upon stimulation as a measure of the host response to infection. Specifically, we calculated the difference of the $\log _{2}$ of expression values between the stimulated and non-stimulated states, corrected for the effect of low values of FPKM, for each gene expressed in at least one of the two conditions.

$$
\begin{aligned}
& \begin{aligned}
\text { Diff } & =\log _{2}\left(1+F P K M_{\text {Stim }}\right)-\log _{2}\left(1+F P K M_{N S}\right) \\
& =\log _{2}\left(\frac{1+F P K M_{\text {Stim }}}{1+F P K M_{N S}}\right)
\end{aligned} \\
& \text { FoldChange }=\frac{1+F P K M_{\text {Stim }}}{1+F P K M_{N S}}=2^{\text {Diff }}
\end{aligned}
$$

For the mapping of eQTMs and reQTMs, we conducted two separate analyses: merging all individuals and including ancestry as a covariate, or keeping the two populations separately.

\section{Expression quantitative trait loci (eQTL) analysis}

We mapped expression quantitative trait loci (eQTLs) using the MatrixEQTL $\mathrm{R}$ package [70], leveraging our genotyping and expression data [48]. As for the meQTL mapping, we filtered out SNPs with a minor allele frequency $<5 \%$ in the populations studied and kept a final dataset of 10,278,745 SNPs. Age and PC1/PC2 of the genotype matrix were used as covariates in the linear model. Two different models were used: merging all individuals and including ancestry as a covariate, or keeping the two populations separately. We also mapped response quantitative trait loci (reQTLs), using the fold change of expression described above, instead of expression, and the same covariates that we used for the eQTL mapping.

For both eQTLs and reQTLs, FDR was computed by mapping eQTLs/reQTLs on 100 datasets with the expression values permuted within each population. We then kept, after each permutation, the most significant $P$ value per gene, across populations (gene-level FDR). Finally, we computed the FDR associated with different $P$ value thresholds for eQTLs or reQTLs, and subsequently selected the $P$ value threshold that provided a 5\% FDR: $P=5 \times 10^{-5}$ and $P=5 \times 10^{-6}$ for eQTLs and reQTLs, respectively.

\section{Simulations to infer causality}

We simulated different scenarios to infer causal relationships between DNA methylation and gene expression. For each scenario, we started by randomly selecting genomic blocks of $1 \mathrm{Mb}$ each along the genome to keep realistic expectations of genetic structure. We next randomly sampled SNPs in these blocks, which we used to simulate methylation and gene expression data. For example, in a scenario where a genetic variant influences DNA methylation variation that, in turn, actively regulates gene expression (see Fig. 4a), we followed the next steps:

(i)

(ii)

$$
G_{i \_s t d}=\frac{\left(G_{i}-\overline{G_{i}}\right)}{s d\left(G_{i}\right)}
$$

$$
M_{i}=\sqrt{\alpha_{i}} \times G_{i_{s t d}}+\sqrt{\left(1-\alpha_{i}\right)} \times \varepsilon_{i}
$$

$$
M_{i \_s t d}=\frac{\left(M_{i}-\overline{M_{i}}\right)}{s d\left(M_{i}\right)}
$$




$$
\begin{aligned}
E_{i}= & \sqrt{\gamma \times \beta_{i}} \times M_{i_{s t d}}+\sqrt{\gamma \times \tau_{i}} \times G_{i_{s t d}} \\
& +\sqrt{\left(1-\gamma \times\left(\beta_{i}+\tau_{i}\right)\right)} \times \zeta_{i}
\end{aligned}
$$

where $G_{i}$ is the genotype of the $i$ th sampled variant and $G_{i \_s t d}$ the standardized value of its genotype; $M_{i}$ is the simulated methylation data and $M_{i_{-} s t d}$ its standardized methylation value; $E_{i}$ is the simulated gene expression data; $\alpha_{i}$ is the proportion of variance of $M_{i}$ that is explained by $G_{i}$, and $\gamma$ is a noise parameter that corresponds to the total proportion of variance of $E_{i}$ that is explained by $G_{i}$ and $M_{i} . \beta_{i}$ and $\tau_{i}$ are the proportions of explained variance that are attributable to $G_{i}$ and $M_{i}$ respectively (satisfying $\beta_{i}+\tau_{i}$ $=1$ ). Finally, $\varepsilon_{i}$ and $\zeta_{i}$ are random, normally distributed residuals. Note that in the simulation presented in Fig. 4a, b, we used a gamma of 0.25 , so that $75 \%$ of the variance of gene expression remained unexplained.

\section{Detection of genetic variants-DNA methylation-gene expression trios}

To infer causality between regulatory loci and gene expression variation, we considered eQTLs that were also detected as meQTLs, and, out of this subset, we kept only those for which the meQTL-CpG had previously been identified as an eQTM of the eQTL-gene (Additional file 1: Figure S11). When multiple SNPs or CpGs where present in a trio, we used an elastic net model, to build linear predictors of (i) gene expression based on DNA methylation variability for trios with multiple CpGs and (ii) DNA methylation based on genetic variability for trios with multiple SNPs. These predictors were then used as summary variables for DNA methylation variability (i) or genetic variability (ii). Specifically, the glmnet function from the $\mathrm{R}$ package glmnet [106] was used to fit the generalized linear model via penalized maximum likelihood, with an elastic net mixing parameter $\alpha$ of 0.5 . The strength of the penalty $\lambda_{1 \mathrm{se}}$ was chosen as the largest value of lambda such that the error was within 1 standard deviation of the minimum lambda, when performing k-fold cross validation with the cv.glmnet function. Finally, the generic $\mathrm{R}$ function predict was used to build the optimal linear predictor in each case. For the trios presenting more than one SNP, we also used a predictor of gene expression based on genetic variability, as summary variable for the genetic variability, and found no differences in our simulation-based mediation results when compared to building the summary variable from a predictor of DNA methylation (data not shown).

\section{Mediation analyses}

For conducting causal mediation analyses, we used a Bayesian approach as implemented in the mediation $\mathrm{R}$ package [90]. Briefly, this approach estimates causal effects of a mediating variable $M$ (DNA methylation) on the relationship between an independent variable $X$ (genetics) and a dependent variable $Y$ (gene expression). In this scenario, the global effect of $X$ on $Y$ can be written as $\rho_{X \rightarrow Y}=\tau+\alpha \cdot \beta$, where $\tau$ is the specific effect of $X$ on $Y, \alpha$ the specific effect of $X$ on $M$, and $\beta$ the specific effect of $M$ on $Y$. With this, the product $\alpha \cdot \beta$ represents the mediation effect of $G$ on $Y$, through $M$. The mediate function of the mediation $\mathrm{R}$ package was used to compute point estimates for average causal mediation effects, as well as 1000 simulation draws of average causal mediation effects. The empirical distribution of simulated effects was used to fit a normal distribution, which was subsequently used to compute empirical $P$ values for the $\mathrm{H}_{0}$ hypothesis " $\alpha \cdot \beta=0$." We used the $\mathrm{R}$ function $p$.adjust with method "fdr" to correct at a FDR $=5 \%$.

For comparison purposes with the mediation analyses, we conducted on simulated data a partial correlation approach to test for independence between expression and methylation levels when accounting for genetic variability. We used the pcor.test function from the R package ppcor [107] to compute $P$ values of the partial correlation between simulated expression and methylation data.

\section{Additional files}

Additional file 1: Figure S1. Overview of the EvolmmunoPop experimental setting. Figure S2. Exploring the non-linear effects of age on DNA methylation. Figure S3. Mono-DMS were detected using the same approach as described in the Materials and methods section, and including the proportions in monocyte subpopulations as covariates. Figure S4. PCA of the genetic data, based on 151,419 SNPs, for Africans (AFB, red dots) and Europeans (EUB, blue dots). Figure S5. Fine mapping of meQTLs. Figure S6. Histogram of physical proximity of cis-meQTLs. Figure S7. Genomic location of CpG sites associated with a meQTL. Figure S8. Proportions of population differences in DNA methylation accounted for by genetics. Figure S9. Fold enrichment of meQTLs in GWAS hits. Figure S10. Fold enrichment of meQTLS associated with DMS in GWAS hits related to "immune system disorder". Figure S11. Rationale for the detection of trios to be used for causality inference. Figure S12. Cartoons of the various simulated scenarios. Figure S13. Heat map of correlation between the first ten PCs of expression and DNA methylation. Figure S14. Genomic location of eQTMS (NS) and reQTMs (for all stimulated conditions). Figure S15. Number of reQTMgenes, per condition, according to the direction of their association with DNA methylation. Figure S16. Causality inference upon immune stimulation. (PDF $3088 \mathrm{~kb}$ )

Additional file 2: Notes 1-2. (PDF $94 \mathrm{~kb}$ )

Additional file 3: Table S1. (XLSX $868 \mathrm{~kb})$

Additional file 4: Table S2. (XLSX $75 \mathrm{~kb}$ )

Additional file 5: Table S3. (XLSX $157 \mathrm{~kb})$

Additional file 6: Table S4. (XLSX $36 \mathrm{~kb})$
Funding

This project was funded by the Institut Pasteur, the CNRS, and the European Research Council under the European Union's Seventh Framework Programme (FP/2007-2013)/ERC grant agreement 281297 (to L.Q.-M.). M.R. was supported by a Marie Skłodowska-Curie fellowship (DLV-655417). 


\section{Availability of data and materials}

The DNA methylation data generated in this study have been deposited in the NCBI Gene Expression Omnibus (GEO) under accession code GSE120610 [108]. Genome-wide SNP genotyping, whole exome sequencing, and RNAsequencing data used in this study are available at the European GenomePhenome Archive (EGA) under accession code EGAS00001001895 [109].

\section{Authors' contributions}

LTH designed and performed the computational analyses, analyzed the data, and interpreted the results, with input from MR, MF, HQ, HA, EP, and LQ-M. LMM, JLM, and MSK contributed the DNA methylation data. NZ contributed the flow cytometry data. MR, HA, and EP contributed with ideas and participated in evaluating results and discussions. LQ-M conceived and supervised the study and obtained the funding. LTH and LQ-M wrote the manuscript, with input from all authors. All authors approved the final manuscript.

\section{Ethics approval and consent to participate}

All healthy donors provided informed consent. All experiments were approved by the Ethics Board of Institut Pasteur (EVOIMMUNOPOP-281297) and the relevant French authorities (CPP, CCITRS and CNIL), subject to applicable laws and regulations and ethical principles consistent with the Declaration of Helsinki.

\section{Competing interests}

The authors declare that they have no competing interests.

\section{Publisher's Note}

Springer Nature remains neutral with regard to jurisdictional claims in published maps and institutional affiliations.

\section{Author details}

${ }^{1}$ Unit of Human Evolutionary Genetics, Institut Pasteur, 75015 Paris, France. ${ }^{2}$ Centre National de la Recherche Scientifique (CNRS) UMR2000, 75015 Paris, France. ${ }^{3}$ Center of Bioinformatics, Biostatistics and Integrative Biology, Institut Pasteur, 75015 Paris, France. ${ }^{4}$ Laboratory for Epigenetics \& Environment, Centre National de Recherche en Génomique Humaine (CNRGH), CEA-Institut de Biologie François Jacob, 91000 Evry, France. ${ }^{5}$ Department of Medical Genetics, University of British Columbia, Centre for Molecular Medicine and Therapeutics, BC Children's Hospital Research Institute, Vancouver, BC, Canada.

\section{Received: 14 June 2018 Accepted: 4 December 2018}

Published online: 18 December 2018

\section{References}

1. Brinkworth JF, Barreiro LB. The contribution of natural selection to presentday susceptibility to chronic inflammatory and autoimmune disease. Curr Opin Immunol. 2014;31:66-78.

2. Casanova JL, Abel L, Quintana-Murci L. Immunology taught by human genetics. Cold Spring Harb Symp Quant Biol. 2013;78:157-72.

3. Casanova JL. Severe infectious diseases of childhood as monogenic inborn errors of immunity. Proc Natl Acad Sci U S A. 2015;112:E7128-37.

4. Casanova JL. Human genetic basis of interindividual variability in the course of infection. Proc Natl Acad Sci U S A. 2015;112:E7118-27.

5. Fumagalli M, Sironi M. Human genome variability, natural selection and infectious diseases. Curr Opin Immunol. 2014;30:9-16.

6. Fumagalli M, Sironi M, Pozzoli U, Ferrer-Admetlla A, Pattini L, Nielsen R. Signatures of environmental genetic adaptation pinpoint pathogens as the main selective pressure through human evolution. PLoS Genet. 2011;7: e1002355

7. Casanova JL, Abel L. Inborn errors of immunity to infection: the rule rather than the exception. J Exp Med. 2005;202:197-201.

8. Karlsson EK, Kwiatkowski DP, Sabeti PC. Natural selection and infectious disease in human populations. Nat Rev Genet. 2014;15:379-93.

9. Quintana-Murci L, Clark AG. Population genetic tools for dissecting innate immunity in humans. Nat Rev Immunol. 2013;13:280-93.

10. Siddle KJ, Quintana-Murci L. The Red Queen's long race: human adaptation to pathogen pressure. Curr Opin Genet Dev. 2014;29:31-8.

11. Barreiro LB, Quintana-Murci L. From evolutionary genetics to human immunology: how selection shapes host defence genes. Nat Rev Genet. 2010;11:17-30
12. Smith ZD, Meissner A. DNA methylation: roles in mammalian development. Nat Rev Genet. 2013;14:204-20.

13. Schubeler D. Function and information content of DNA methylation. Nature. 2015;517:321-6.

14. Feil R, Fraga MF. Epigenetics and the environment: emerging patterns and implications. Nat Rev Genet. 2011;13:97-109.

15. Kaminsky ZA, Tang T, Wang SC, Ptak C, Oh GH, Wong AH, Feldcamp LA, Virtanen C, Halfvarson J, Tysk C, et al. DNA methylation profiles in monozygotic and dizygotic twins. Nature Genet. 2009;41:240-5.

16. Lam LL, Emberly E, Fraser HB, Neumann SM, Chen E, Miller GE, Kobor MS. Factors underlying variable DNA methylation in a human community cohort. Proc Natl Acad Sci U S A. 2012;109(Suppl 2):17253-60.

17. Ziller MJ, Gu H, Muller F, Donaghey J, Tsai LT, Kohlbacher O, De Jager PL, Rosen ED, Bennett DA, Bernstein BE, et al. Charting a dynamic DNA methylation landscape of the human genome. Nature. 2013;500:477-81.

18. Marr AK, Maclsaac JL, Jiang R, Airo AM, Kobor MS, McMaster WR. Leishmania donovani infection causes distinct epigenetic DNA methylation changes in host macrophages. PLoS Pathog. 2014;10:e1004419.

19. Pacis A, Tailleux L, Morin AM, Lambourne J, Maclsaac JL, Yotova V, Dumaine A, Danckaert A, Luca F, Grenier JC, et al. Bacterial infection remodels the DNA methylation landscape of human dendritic cells. Genome Res. 2015;25: 1801-11.

20. Gibbs JR, van der Brug MP, Hernandez DG, Traynor BJ, Nalls MA, Lai SL, Arepalli S, Dillman A, Rafferty IP, Troncoso J, et al. Abundant quantitative trait loci exist for DNA methylation and gene expression in human brain. PLoS Genet. 2010;6:e1000952

21. Zhang D, Cheng L, Badner JA, Chen C, Chen Q, Luo W, Craig DW, Redman M, Gershon ES, Liu C. Genetic control of individual differences in genespecific methylation in human brain. Am J Hum Genet. 2010;86:411-9.

22. Bell JT, Pai AA, Pickrell JK, Gaffney DJ, Pique-Regi R, Degner JF, Gilad Y, Pritchard JK. DNA methylation patterns associate with genetic and gene expression variation in HapMap cell lines. Genome Biol. 2011;12:R10.

23. Gutierrez-Arcelus M, Lappalainen T, Montgomery SB, Buil A, Ongen H, Yurovsky A, Bryois J, Giger T, Romano L, Planchon A, et al. Passive and active DNA methylation and the interplay with genetic variation in gene regulation. eLife. 2013;2:e00523.

24. Banovich NE, Lan X, McVicker G, van de Geijn B, Degner JF, Blischak JD, Roux J, Pritchard JK, Gilad Y. Methylation QTLs are associated with coordinated changes in transcription factor binding, histone modifications, and gene expression levels. PLoS Genet. 2014;10:e1004663.

25. Olsson AH, Volkov P, Bacos K, Dayeh T, Hall E, Nilsson EA, Ladenvall C, Ronn $\mathrm{T}$, Ling C. Genome-wide associations between genetic and epigenetic variation influence mRNA expression and insulin secretion in human pancreatic islets. PLoS Genet. 2014;10:e1004735.

26. Hannon E, Dempster E, Viana J, Burrage J, Smith AR, Macdonald R, St Clair D, Mustard C, Breen G, Therman S, et al. An integrated genetic-epigenetic analysis of schizophrenia: evidence for co-localization of genetic associations and differential DNA methylation. Genome Biol. 2016;17:176.

27. Hannon E, Spiers H, Viana J, Pidsley R, Burrage J, Murphy TM, Troakes C, Turecki G, O'Donovan MC, Schalkwyk LC, et al. Methylation QTLs in the developing brain and their enrichment in schizophrenia risk loci. Nat Neurosci. 2016;19:48-54.

28. Wagner JR, Busche $\mathrm{S}, \mathrm{Ge} B$, Kwan T, Pastinen T, Blanchette M. The relationship between DNA methylation, genetic and expression interindividual variation in untransformed human fibroblasts. Genome Biol. 2014; 15:R37.

29. van Dongen J, Nivard MG, Willemsen G, Hottenga JJ, Helmer Q, Dolan CV, Ehli EA, Davies GE, van Iterson M, Breeze CE, et al. Genetic and environmental influences interact with age and sex in shaping the human methylome. Nat Commun. 2016;7:11115.

30. McClay JL, Shabalin AA, Dozmorov MG, Adkins DE, Kumar G, Nerella S, Clark SL, Bergen SE, Swedish Schizophrenia C, Hultman CM, et al. High density methylation QTL analysis in human blood via next-generation sequencing of the methylated genomic DNA fraction. Genome Biol. 2015;16:291.

31. Bell JT, Tsai PC, Yang TP, Pidsley R, Nisbet J, Glass D, Mangino M, Zhai G, Zhang F, Valdes A, et al. Epigenome-wide scans identify differentially methylated regions for age and age-related phenotypes in a healthy ageing population. PLoS Genet. 2012;8:e1002629.

32. Wahl S, Drong A, Lehne B, Loh M, Scott WR, Kunze S, Tsai PC, Ried JS, Zhang $W$, Yang $Y$, et al. Epigenome-wide association study of body mass index, and the adverse outcomes of adiposity. Nature. 2017;541:81-6. 
33. Kulis M, Esteller M. DNA methylation and cancer. Adv Genet. 2010;70:27-56.

34. Baylin SB, Jones PA. Epigenetic determinants of cancer. Cold Spring Harb Perspect Biol. 2016;8(9):a019505.

35. Bell CG, Finer S, Lindgren CM, Wilson GA, Rakyan VK, Teschendorff AE, Akan P, Stupka E, Down TA, Prokopenko I, et al. Integrated genetic and epigenetic analysis identifies haplotype-specific methylation in the FTO type 2 diabetes and obesity susceptibility locus. PLoS One. 2010;5:e14040.

36. Chambers JC, Loh M, Lehne B, Drong A, Kriebel J, Motta V, Wahl S, Elliott HR, Rota F, Scott WR, et al. Epigenome-wide association of DNA methylation markers in peripheral blood from Indian Asians and Europeans with incident type 2 diabetes: a nested case-control study. Lancet Diabetes Endocrinol. 2015;3:526-34.

37. Liu Y, Aryee MJ, Padyukov L, Fallin MD, Hesselberg E, Runarsson A, Reinius L, Acevedo N, Taub M, Ronninger M, et al. Epigenome-wide association data implicate DNA methylation as an intermediary of genetic risk in rheumatoid arthritis. Nat Biotechnol. 2013;31:142-7.

38. Heyn H, Moran S, Hernando-Herraez I, Sayols S, Gomez A, Sandoval J, Monk D, Hata K, Marques-Bonet T, Wang L, Esteller M. DNA methylation contributes to natural human variation. Genome Res. 2013;23:1363-72.

39. Moen EL, Zhang X, Mu W, Delaney SM, Wing C, McQuade J, Myers J, Godley LA, Dolan ME, Zhang W. Genome-wide variation of cytosine modifications between European and African populations and the implications for complex traits. Genetics. 2013;194:987-96.

40. Fraser HB, Lam LL, Neumann SM, Kobor MS. Population-specificity of human DNA methylation. Genome Biol. 2012;13:R8.

41. Fagny M, Patin E, Maclsaac JL, Rotival M, Flutre T, Jones MJ, Siddle KJ, Quach H, Harmant C, McEwen LM, et al. The epigenomic landscape of African rainforest hunter-gatherers and farmers. Nat Commun. 2015;6:10047.

42. Carja O, Maclsaac JL, Mah SM, Henn BM, Kobor MS, Feldman MW, Fraser HB. Worldwide patterns of human epigenetic variation. Nat Ecol Evol. 2017;1: 1577-83.

43. Galanter JM, Gignoux CR, Oh SS, Torgerson D, Pino-Yanes M, Thakur N, Eng C, Hu D, Huntsman S, Farber HJ, et al. Differential methylation between ethnic sub-groups reflects the effect of genetic ancestry and environmental exposures. Elife. 2017;6:e20532.

44. Gopalan S, Carja O, Fagny M, Patin E, Myrick JW, McEwen LM, Mah SM, Kobor MS, Froment A, Feldman MW, et al. Trends in DNA methylation with age replicate across diverse human populations. Genetics. 2017; 206:1659-74.

45. Sugawara H, Iwamoto K, Bundo M, Ueda J, Ishigooka J, Kato T. Comprehensive DNA methylation analysis of human peripheral blood leukocytes and lymphoblastoid cell lines. Epigenetics. 2011;6:508-15.

46. Houseman EA, Accomando WP, Koestler DC, Christensen BC, Marsit CJ, Nelson HH, Wiencke JK, Kelsey KT. DNA methylation arrays as surrogate measures of cell mixture distribution. BMC Bioinformatics. 2012;13:86.

47. Teschendorff AE, Relton CL. Statistical and integrative system-level analysis of DNA methylation data. Nat Rev Genet. 2018;19:129-47.

48. Quach H, Rotival M, Pothlichet J, Loh YE, Dannemann M, Zidane N, Laval G, Patin E, Harmant C, Lopez M, et al. Genetic adaptation and Neandertal admixture shaped the immune system of human populations. Cell. 2016; 167:643-56 e617.

49. Nedelec Y, Sanz J, Baharian G, Szpiech ZA, Pacis A, Dumaine A, Grenier JC, Freiman A, Sams AJ, Hebert S, et al. Genetic ancestry and natural selection drive population differences in immune responses to pathogens. Cell. 2016; 167:657-69 e621.

50. Barreiro LB, Tailleux L, Pai AA, Gicquel B, Marioni JC, Gilad Y. Deciphering the genetic architecture of variation in the immune response to Mycobacterium tuberculosis infection. Proc Natl Acad Sci U S A. 2012;109:1204-9.

51. Fairfax BP, Humburg $P$, Makino S, Naranbhai V, Wong D, Lau E, Jostins $L$, Plant K, Andrews R, McGee C, Knight JC. Innate immune activity conditions the effect of regulatory variants upon monocyte gene expression. Science. 2014;343:1246949.

52. Lee MN, Ye C, Villani AC, Raj T, Li W, Eisenhaure TM, Imboywa SH, Chipendo PI, Ran FA, Slowikowski K, et al. Common genetic variants modulate pathogen-sensing responses in human dendritic cells. Science. 2014;343: 1246980.

53. Caliskan M, Baker SW, Gilad Y, Ober C. Host genetic variation influences gene expression response to rhinovirus infection. PLoS Genet. 2015;11: e1005111.

54. Kim S, Becker J, Bechheim M, Kaiser V, Noursadeghi M, Fricker N, Beier E, Klaschik S, Boor P, Hess T, et al. Characterizing the genetic basis of innate immune response in TLR4-activated human monocytes. Nat Commun. 2014; 5:5236.

55. Kim-Hellmuth S, Bechheim M, Putz B, Mohammadi P, Nedelec Y, Giangreco $N$, Becker J, Kaiser V, Fricker N, Beier E, et al. Genetic regulatory effects modified by immune activation contribute to autoimmune disease associations. Nat Commun. 2017:8:266.

56. Pai AA, Pritchard JK, Gilad Y. The genetic and mechanistic basis for variation in gene regulation. PLoS Genet. 2015;11:e1004857.

57. Yang X, Han H, De Carvalho DD, Lay FD, Jones PA, Liang G. Gene body methylation can alter gene expression and is a therapeutic target in cancer. Cancer Cell. 2014;26:577-90.

58. Jjingo $\mathrm{D}$, Conley $A B, Y$, SV, Lunyak W, Jordan IK. On the presence and role of human gene-body DNA methylation. Oncotarget. 2012;3:462-74.

59. Maunakea AK, Nagarajan RP, Bilenky M, Ballinger TJ, D'Souza C, Fouse SD, Johnson BE, Hong C, Nielsen C, Zhao Y, et al. Conserved role of intragenic DNA methylation in regulating alternative promoters. Nature. 2010;466:253-7.

60. Gutierrez-Arcelus M, Ongen H, Lappalainen T, Montgomery SB, Buil A, Yurovsky A, Bryois J, Padioleau I, Romano L, Planchon A, et al. Tissue-specific effects of genetic and epigenetic variation on gene regulation and splicing. PLoS Genet. 2015:11:e1004958.

61. Relton CL, Davey Smith G. Two-step epigenetic Mendelian randomization: a strategy for establishing the causal role of epigenetic processes in pathways to disease. Int J Epidemiol. 2012;41:161-76.

62. Richardson TG, Zheng J, Davey Smith G, Timpson NJ, Gaunt TR, Relton CL, Hemani G. Mendelian randomization analysis identifies CpG sites as putative mediators for genetic influences on cardiovascular disease risk. Am J Hum Genet. 2017;101:590-602.

63. Hannon E, Weedon M, Bray N, O'Donovan M, Mill J. Pleiotropic effects of trait-associated genetic variation on DNA methylation: utility for refining GWAS loci. Am J Hum Genet. 2017;100:954-9.

64. Bell CG, Gao F, Yuan W, Roos L, Acton RJ, Xia Y, Bell J, Ward K, Mangino M, Hysi $P G$, et al. Obligatory and facilitative allelic variation in the DNA methylome within common disease-associated loci. Nat Commun. 2018;9:8.

65. Bonder MJ, Luijk R, Zhernakova DV, Moed M, Deelen P, Vermaat M, van Iterson $M$, van Dijk F, van Galen M, Bot J, et al. Disease variants alter transcription factor levels and methylation of their binding sites. Nat Genet. 2017;49:131-8.

66. Pickrell JK. Joint analysis of functional genomic data and genome-wide association studies of 18 human traits. Am J Hum Genet. 2014;94:559-73.

67. Schaub MA, Boyle AP, Kundaje A, Batzoglou S, Snyder M. Linking disease associations with regulatory information in the human genome. Genome Res. 2012;22:1748-59.

68. Du P, Zhang X, Huang CC, Jafari N, Kibbe WA, Hou L, Lin SM. Comparison of Beta-value and M-value methods for quantifying methylation levels by microarray analysis. BMC Bioinformatics. 2010;11:587.

69. Johnson ND, Wiener HW, Smith AK, Nishitani S, Absher DM, Arnett DK, Aslibekyan S, Conneely KN. Non-linear patterns in age-related DNA methylation may reflect CD4(+) T cell differentiation. Epigenetics. 2017; 12:492-503.

70. Shabalin AA. Matrix eQTL: ultra fast eQTL analysis via large matrix operations. Bioinformatics. 2012;28:1353-8.

71. Emilsson V, Thorleifsson G, Zhang B, Leonardson AS, Zink F, Zhu J, Carlson S, Helgason A, Walters GB, Gunnarsdottir S, et al. Genetics of gene expression and its effect on disease. Nature. 2008;452:423-8.

72. Dermitzakis ET. Cellular genomics for complex traits. Nat Rev Genet. 2012;13: 215-20.

73. Nica AC, Montgomery SB, Dimas AS, Stranger BE, Beazley C, Barroso I, Dermitzakis ET. Candidate causal regulatory effects by integration of expression QTLs with complex trait genetic associations. PLoS Genet. 2010; 6:e1000895.

74. Cookson W, Liang L, Abecasis G, Moffatt M, Lathrop M. Mapping complex disease traits with global gene expression. Nat Rev Genet. 2009;10:184-94.

75. Malone J, Holloway E, Adamusiak T, Kapushesky M, Zheng J, Kolesnikov N, Zhukova A, Brazma A, Parkinson H. Modeling sample variables with an Experimental Factor Ontology. Bioinformatics. 2010;26:1112-8.

76. Gu J, Stevens M, Xing X, Li D, Zhang B, Payton JE, Oltz EM, Jarvis JN, Jiang K, Cicero $T$, et al. Mapping of variable DNA methylation across multiple cell types defines a dynamic regulatory landscape of the human genome. G3 (Bethesda). 2016;6:973-86.

77. Hannon E, Lunnon K, Schalkwyk L, Mill J. Interindividual methylomic variation across blood, cortex, and cerebellum: implications for epigenetic 
studies of neurological and neuropsychiatric phenotypes. Epigenetics. 2015; 10:1024-32.

78. Cheung WA, Shao X, Morin A, Siroux V, Kwan T, Ge B, Aissi D, Chen L, Vasquez $L$, Allum F, et al. Functional variation in allelic methylomes underscores a strong genetic contribution and reveals novel epigenetic alterations in the human epigenome. Genome Biol. 2017;18:50.

79. Schultz MD, He Y, Whitaker JW, Hariharan M, Mukamel EA, Leung D, Rajagopal $\mathrm{N}$, Nery JR, Urich MA, Chen $\mathrm{H}$, et al. Human body epigenome maps reveal noncanonical DNA methylation variation. Nature. 2015;523:212-6.

80. Farre P, Jones MJ, Meaney MJ, Emberly E, Turecki G, Kobor MS. Concordant and discordant DNA methylation signatures of aging in human blood and brain. Epigenetics Chromatin. 2015;8:19.

81. Pai AA, Bell JT, Marioni JC, Pritchard JK, Gilad Y. A genome-wide study of DNA methylation patterns and gene expression levels in multiple human and chimpanzee tissues. PLoS Genet. 2011;7:e1001316.

82. Nititham J, Taylor KE, Gupta R, Chen H, Ahn R, Liu J, Seielstad M, Ma A, Bowcock AM, Criswell LA, et al. Meta-analysis of the TNFAIP3 region in psoriasis reveals a risk haplotype that is distinct from other autoimmune diseases. Genes Immun. 2015;16:120-6.

83. Yin X, Low HQ, Wang L, Li Y, Ellinghaus E, Han J, Estivill X, Sun L, Zuo X, Shen $C$, et al. Genome-wide meta-analysis identifies multiple novel associations and ethnic heterogeneity of psoriasis susceptibility. Nat Commun. 2015;6:6916.

84. Bonder MJ, Kasela S, Kals M, Tamm R, Lokk K, Barragan I, Buurman WA, Deelen $\mathrm{P}$, Greve JW, Ivanov M, et al. Genetic and epigenetic regulation of gene expression in fetal and adult human livers. BMC Genomics. 2014;15:860.

85. Ecker S, Chen L, Pancaldi V, Bagger FO, Fernandez JM, Carrillo de Santa Pau E, Juan D, Mann AL, Watt S, Casale FP, et al. Genome-wide analysis of differential transcriptional and epigenetic variability across human immune cell types. Genome Biol. 2017;18:18.

86. Shlyueva D, Stampfel G, Stark A. Transcriptional enhancers: from properties to genome-wide predictions. Nat Rev Genet. 2014;15:272-86.

87. Rickels R, Shilatifard A. Enhancer logic and mechanics in development and disease. Trends Cell Biol. 2018;28(8):608-30.

88. Yao L, Berman BP, Farnham PJ. Demystifying the secret mission of enhancers: linking distal regulatory elements to target genes. Crit Rev Biochem Mol Biol. 2015;50:550-73.

89. Grundberg E, Meduri E, Sandling JK, Hedman AK, Keildson S, Buil A, Busche S, Yuan W, Nisbet J, Sekowska M, et al. Global analysis of DNA methylation variation in adipose tissue from twins reveals links to disease-associated variants in distal regulatory elements. Am J Hum Genet. 2013;93:876-90.

90. Tingley D, Yamamoto T, Hirose K, Keele L, Imai K. mediation: R package for causal mediation analysis. J Stat Softw. 2014;59:1-38.

91. MacKinnon DP. Multivariate applications series. Introduction to statistical mediation analysis. New York: Taylor \& Francis Group/Lawrence Erlbaum Associates; 2008.

92. Stadler MB, Murr R, Burger L, Ivanek R, Lienert F, Scholer A, van Nimwegen E, Wirbelauer C, Oakeley EJ, Gaidatzis D, et al. DNA-binding factors shape the mouse methylome at distal regulatory regions. Nature. 2011;480:490-5.

93. Stroud H, Feng S, Morey Kinney S, Pradhan S, Jacobsen SE. 5Hydroxymethylcytosine is associated with enhancers and gene bodies in human embryonic stem cells. Genome Biol. 2011;12:R54.

94. Choi I, Kim R, Lim HW, Kaestner KH, Won KJ. 5-Hydroxymethylcytosine represses the activity of enhancers in embryonic stem cells: a new epigenetic signature for gene regulation. BMC Genomics. 2014;15:670.

95. Song MA, Brasky TM, Marian C, Weng DY, Taslim C, Dumitrescu RG, Llanos AA, Freudenheim $J$, Shields PG. Racial differences in genome-wide methylation profiling and gene expression in breast tissues from healthy women. Epigenetics. 2015;10:1177-87.

96. Zaret KS, Carroll JS. Pioneer transcription factors: establishing competence for gene expression. Genes Dev. 2011;25:2227-41.

97. Valeri L, Reese SL, Zhao S, Page CM, Nystad W, Coull BA, London SJ. Misclassified exposure in epigenetic mediation analyses. Does DNA methylation mediate effects of smoking on birthweight? Epigenomics. 2017; 9:253-65.

98. Wang F, Zhang S, Wen Y, Wei Y, Yan H, Liu H, Su J, Zhang Y, Che J. Revealing the architecture of genetic and epigenetic regulation: a maximum likelihood model. Brief Bioinform. 2014;15:1028-43.

99. Genomes Project C, Auton A, Brooks LD, Durbin RM, Garrison EP, Kang HM, Korbel JO, Marchini JL, McCarthy S, McVean GA, Abecasis GR. A global reference for human genetic variation. Nature. 2015;526:68-74.
100. Du P, Kibbe WA, Lin SM. lumi: a pipeline for processing Illumina microarray. Bioinformatics. 2008;24:1547-8

101. Aryee MJ, Jaffe AE, Corrada-Bravo H, Ladd-Acosta C, Feinberg AP, Hansen $K D$, Irizarry RA. Minfi: a flexible and comprehensive Bioconductor package for the analysis of Infinium DNA methylation microarrays. Bioinformatics. 2014;30:1363-9.

102. Teschendorff AE, Marabita F, Lechner M, Bartlett T, Tegner J, Gomez-Cabrero D, Beck S. A beta-mixture quantile normalization method for correcting probe design bias in Illumina Infinium 450 k DNA methylation data. Bioinformatics. 2013;29:189-96.

103. Leek JT, Johnson WE, Parker HS, Jaffe AE, Storey JD. The sva package for removing batch effects and other unwanted variation in high-throughput experiments. Bioinformatics. 2012;28:882-3.

104. Smyth GK. Limma: linear models for microarray data. In: Gentleman R, Carey V, Dudoit S, Irizarry I, Hube W, editors. Bioinformatics and computational biology solutions using R and Bioconductor. New York: Springer; 2005. p. 397-420.

105. MacArthur J, Bowler E, Cerezo M, Gil L, Hall P, Hastings E, Junkins H, McMahon A, Milano A, Morales J, et al. The new NHGRI-EBI Catalog of published genome-wide association studies (GWAS Catalog). Nucleic Acids Res. 2017;45:D896-901.

106. Friedman J, Hastie T, Tibshirani R. Regularization paths for generalized linear models via coordinate descent. J Stat Softw. 2010;33:1-22.

107. Kim S. ppcor: an R package for a fast calculation to semi-partial correlation coefficients. Commun Stat Appl Methods. 2015;22:665-74.

108. Husquin LT, Rotival M, Fagny M, et al. Exploring the genetic basis of human population differences in DNA methylation and their causal impact on immune gene regulation. GEO. 2018; https://www.ncbi.nlm.nih.gov/geo/ query/acc.cgi?acc=GSE120610. Accessed 30 Oct 2018.

109. Quach H, Rotival M, Pothlichet J, et al. Genetic adaptation and Neandertal admixture shaped the immune system of human populations. EGA. 2016; https://www.ebi.ac.uk/ega/studies/EGAS00001001895. Accessed 20 Oct 2016

110. Ernst J, Kellis M. ChromHMM: automating chromatin-state discovery and characterization. Nat Methods. 2012;9:215-6.

111. Ernst J, Kellis M. Chromatin-state discovery and genome annotation with ChromHMM. Nat Protoc. 2017:12:2478-92.

\section{Ready to submit your research? Choose BMC and benefit from:}

- fast, convenient online submission

- thorough peer review by experienced researchers in your field

- rapid publication on acceptance

- support for research data, including large and complex data types

- gold Open Access which fosters wider collaboration and increased citations

- maximum visibility for your research: over $100 \mathrm{M}$ website views per year

At BMC, research is always in progress.

Learn more biomedcentral.com/submissions 